KCL-MTH-99-46

LAPTH-778/2000

LTH-466

hep-th/0001138

August 22, 2018

\title{
Four-point functions in $N=2$ superconformal field theories
}

\author{
B. Eden ${ }^{a}$, P.S. Howe ${ }^{b}$, A. Pickering ${ }^{c}$, E. Sokatchev ${ }^{a}$ and P.C. West ${ }^{b}$ \\ ${ }^{b}$ Department of Mathematics, King's College, London, UK \\ ${ }^{a}$ Laboratoire d'Annecy-le-Vieux de Physique Théoriqu日 LAPTH, Chemin de Bellevue, B.P. 110, \\ F-74941 Annecy-le-Vieux, France \\ c Division of Theoretical Physics, Department of Mathematical Sciences, University of Liverpool, UK
}

\begin{abstract}
Four-point correlation functions of hypermultiplet bilinear composites are analysed in $N=$ 2 superconformal field theory using the superconformal Ward identities and the analyticity properties of the composite operator superfields. It is shown that the complete amplitude is determined by a single arbitrary function of the two conformal cross-ratios of the space-time variables.
\end{abstract}

\footnotetext{
${ }^{1}$ UMR 5108 associée à l'Université de Savoie
} 


\section{Introduction}

In this paper we examine in detail the constraints imposed by superconformal invariance and analyticity on four-point correlation functions of analytic operators in four-dimensional superconformal field theories with $N=2$ supersymmetry. The analytic operators under consideration are gauge-invariant products of hypermultiplets which are represented by analytic superfields in $N=2$ harmonic superspace. Analytic superfields obey a generalised chirality-type constraint and depend holomorphically on the coordinates of the two-sphere which is adjoined to Minkowski superspace to form harmonic superspace. The definition of analyticity is given in more detail below.

The sphere can be thought of as the homogeneous space $U(1) \backslash S U(2)$ and fields carry a charge with respect to the $U(1)$ isotropy group of this internal space. We shall be particularly interested in the case where each of the operators has charge 2 . These operators are hypermultiplet bilinears and also have dimension 2. For the particular case of $N=4$ super Yang-Mills theory (SYM) there is a single hypermultiplet transforming under the adjoint representation of the gauge group and from this and its conjugate one can construct three charge 2 analytic bilinears. They can be viewed as $N=2$ components of the $N=4$ supercurrent, so that the corresponding fourpoint correlation functions give $N=2$ projections of the $N=4$ four-point correlator of four supercurrents. This correlator, or particular spacetime components of it, has been much studied in the context of the Maldacena conjecture [1] on both the AdS [2] and field theory sides [3, 4].

The study of correlation functions of the above type in the harmonic superspace setting has been advocated in a series of papers 15, 6/ 2. The exact functional forms for two- and three-point functions were given [5, 8] (see also [9, 10, 11]) and it was later argued that the coefficients of such correlators in $N=4$ should be non-renormalised [21] using analytic superspace techniques, the reduction formula and the notion of $U(1)_{Y}$ symmetry introduced in 12]. (For the supercurrent correlators this non-renormalisation can also be seen as a consequence of anomaly considerations [10, 8].) It was also conjectured that four-point correlators of operators with sufficiently low charges might be soluble [5, 6. However, in spite of the claims made for future work in these references, this turns out not to be the case and one purpose of the present work is to give the precise result that one finds for four charge 2 operators, which are known to be non-trivial [3, 4]. As we shall show, the requirement of analyticity leads to additional constraints beyond those that one might expect on grounds of superconformal symmetry alone, but these are not enough to determine the correlation function completely. Elsewhere the result for charge 2 operators has been used to simplify the computation of the four-point function at two loops in perturbation theory [13].

The work described here has been carried out over a period of several years and has focused on four-point functions of operators with equal charges. As we have just mentioned, these results are not as strong as had been conjectured. More recently, however, it has become apparent that more striking results can be obtained by considering asymmetric sets of charges. In [14 the methods of the present paper were used to show that four-point extremal correlators $\left(p_{4}=p_{1}+p_{2}+p_{3}, p_{i}=\right.$ charges $)$ are simply given by products of free two-point functions. A discussion in $N=1$ perturbation theory is given in [17]. Since the analogous result had already been found in AdS supergravity 15 (see also 16]), this also established a part of the Maldacena conjecture. So, although the initial conjectures of [5, 6 ] for equal charges have turned out to be incorrect, perhaps it is not unreasonable to claim some partial vindication for the initial optimism in the light of the fact that the very same strategy gives a good way of proving the recent results for extremal correlators in a field-theoretic setting.

The analysis of analytic operators and correlation functions can be carried out in two equivalent frameworks each having their own distinctive features. One can either work in analytic superspace (with complexified spacetime) using explicit coordinates, or one can work in harmonic

\footnotetext{
${ }^{2}$ For an analysis of superconformal field theories in Minkowski superspace see, for example, $(7$ )
} 
superspace (with real spacetime) using an equivariant formalism with respect to the internal $S U(2)$ symmetry group. In the former approach, all the coordinates appear on an equal footing. This makes the action of the superconformal group is more transparent and facilitates the construction of invariants. Further, analyticity (or the lack of it) is manifest. In the latter approach the internal $S U(2)$ is treated covariantly, so that explicit coordinates for the sphere do not have to be introduced. In addition, harmonic analyticity can be interpreted as an irreducibility condition under $S U(2)$. This allows one to limit the analysis to the first two non-trivial levels in the $\theta$ expansion of the correlator. We shall employ both methods in this paper, using the analytic formalism in section 2 and the covariant formulation in section 3.

\section{Coordinate approach}

\subsection{Analytic superspace}

$N=2$ harmonic superspace, first introduced in [18], is the product of $N=2$ Minkowski superspace and the two-sphere $S^{2}=\mathbb{C} P^{1}$. A field on this space can be expanded in harmonics on the sphere with coefficients which are ordinary $N=2$ superfields. A general superfield on $N=2$ harmonic superspace is therefore equivalent to an infinite number of ordinary superfields, but, if a superfield depends holomorphically on the coordinates of the sphere, it will have a finite expansion in ordinary superfields due to the fact that spaces of holomorphic tensors on the sphere are finite-dimensional. Such a field is called harmonic analytic (H-analytic). In addition one can define a generalised notion of chirality, called Grassmann analyticity (G-analyticity) such that a G-analytic field depends on only half the number of odd coordinates of the full superspace. A field which is both $\mathrm{H}$-analytic and $\mathrm{G}$-analytic will be called analytic.

An alternative description of analytic superfields on complexified Minkowski superspace is in terms of (holomorphic) fields on analytic superspace, a space with half the odd dimensionality of harmonic superspace. It bears a similar relation to harmonic superspace as chiral superspace does to Minkowski superspace.

Analytic superspace is a homogeneous space of the complexified $N=2$ superconformal group, $S L(4 \mid 2)$ (for a review of various homogeneous superspaces in this context see [19]). This group acts naturally (to the left) on $N=2$ supertwistor space $\mathbb{C}^{4 \mid 2}$, and the coset we are interested in has the geometrical interpretation as the Grassmannian of (2|1)-planes in twistor space. The body of the whole of this Grassmannian is compact, whereas we are interested only in the usual, non-compact Minkowski spacetime. The body of the actual space we shall work with will therefore be restricted in this sense, although its internal part, $\mathbb{C} P^{1}$, remains compact. This is similar in spirit to regarding $\mathbb{C}$ as an open subset of $\mathbb{C} P^{1}$ obtained by omitting the point at infinity. We shall be a bit more precise about this below, after we have introduced appropriate local coordinates.

In the usual basis for $N=2$ supertwistor space the first four elements correspond to the even part and the second two to the odd part. If we instead make a choice of basis ordered in the sequence two even, one odd, two even, one odd, the isotropy group, $H$, of analytic superspace will consist of supermatrices of the simple form

$$
\left(\begin{array}{ll}
\bullet & 0 \\
\bullet & \bullet
\end{array}\right)
$$

Here each entry represents a $(2 \mid 1) \times(2 \mid 1)$ supermatrix and the bullets denote non-singular such matrices. The superdeterminant is constrained to be 1 . In this basis it is reasonably clear that the coset space $H \backslash S L(4 \mid 2)$ is indeed the Grassmannian of (2|1)-planes in $\mathbb{C}^{4 \mid 2}$. 
Using standard homogenous space techniques we may choose a local coset representative $s(X), X \in$ $M_{A}$ (analytic superspace) as follows:

$$
M_{A} \ni X \rightarrow s(X)=\left(\begin{array}{cc}
1 & X \\
0 & 1
\end{array}\right) \in S L(4 \mid 2)
$$

and again each entry represents a $(2 \mid 1) \times(2 \mid 1)$ supermatrix. The components of $X$ are given by

$$
X=\left(\begin{array}{cc}
x^{\alpha \dot{\alpha}} & \lambda^{\alpha} \\
\pi^{\dot{\alpha}} & y
\end{array}\right)
$$

where $\alpha, \dot{\alpha}$ are two-component spinor indices, $x$ is the spacetime coordinate, $\lambda$ and $\pi$ are the odd coordinates and $y$ is the standard coordinate on $\mathbb{C} P^{1}$. (We shall occasionally use index notation $X^{A A^{\prime}}$ for the coordinate matrix $X$.) As we mentioned above, we want the body of our superspace to consist of non-compact spacetime together with a compact internal space, $\mathbb{C} P^{1}$. The full space we are interested in can be covered by two open sets corresponding to the two standard open sets of the sphere. If we denote these two sets by $U$ and $U^{\prime}$, and put primes on the coordinates for $U^{\prime}$ we find that the two sets are related as follows on the overlap:

$$
\begin{aligned}
x^{\prime} & =x-\frac{\lambda \pi}{y}, \\
\lambda^{\prime} & =\frac{1}{y} \lambda, \\
\pi^{\prime} & =\frac{1}{y} \pi, \\
y^{\prime} & =\frac{1}{y} .
\end{aligned}
$$

We see that the odd coordinates and the coordinates of the internal space together parametrise $\mathbb{C}^{(1 \mid 4)}$, so that the whole space has the form of an affine bundle of rank $(4 \mid 0)$ over $\mathbb{C} P^{(1 \mid 4)}$.

Superconformal transformations can be discussed straightforwardly using standard homogeneous space methods. Under a superconformal transformation $X \rightarrow X \cdot g, g \in S L(4 \mid 2)$. The transformed coordinates $X \cdot g$ are determined using the formula

$$
s(X \cdot g)=h(X, g) s(X) g .
$$

The rôle of the compensating transform $h(X, g)$ (an element of $H$ ) is to restore the form of the coset representative $s(X)$ after right-multiplication by $g$. For an infinitesimal transformation specified by $\mathcal{A} \in \mathfrak{s l}(4 \mid 2)$, the Lie superalgebra of $S L(4 \mid 2)$, we have

$$
\delta X=B+A X+X D+X C X
$$

with

$$
\mathcal{A}=\left(\begin{array}{ll}
-A & B \\
-C & D
\end{array}\right) .
$$

An analytic field of charge $p$ is a field $\phi$ on analytic superspace which transforms under an infinitesimal superconformal transformation according to the rule 


$$
\delta \phi=V \phi+p \Delta \phi
$$

where $V$ is the vector field generating the transformation, $V=\delta X \frac{\partial}{\partial X}$, and $\Delta:=\operatorname{str}(A+X C)$. The free, on-shell hypermultiplet is such a field with charge 1 and will be discussed in more detail below. In an interacting theory, the on-shell hypermultiplet is covariantly analytic, but gauge-invariant products of hypermultiplets are analytic in the above sense with charges equal to the number of hypermultiplets in the product.

For completeness we reproduce here the explicit expressions for the vector fields corresponding to the different types of superconformal transformation. From (6) one can read off the vector fields for each of the parameters. They divide into translational $(B)$, linear $(A, D)$ and quadratic $(C)$ types. The translations are ordinary spacetime translations, half of the $Q$-supersymmetry transformations and translations in the internal $y$ space, $\mathbb{C} P^{1}$. The corresponding vector fields are

$$
V_{A A^{\prime}}=\frac{\partial}{\partial X^{A A^{\prime}}}
$$

The linearly realised symmetries are Lorentz transformations $(S L(2) \times S L(2))$ in complex spacetime) and dilations, internal dilations, $R$-symmetry transformations, the other half of the $Q$ supersymmetries and half of the $S$-supersymmetries. The Lorentz transformations are handled in the usual way so that we do not need to write them down. The vector fields generating dilations $(D)$, internal dilations $\left(D^{\prime}\right)$ and $R$-symmetry transformations are

$$
\begin{aligned}
V(D) & =x^{\alpha \dot{\alpha}} \partial_{\alpha \dot{\alpha}}+\frac{1}{2}\left(\lambda^{\alpha^{\prime}} \partial_{\alpha}+\pi^{\dot{\alpha}} \partial_{\dot{\alpha}}\right), \\
V\left(D^{\prime}\right) & =y \partial_{y}+\frac{1}{2}\left(\lambda^{\alpha} \partial_{\alpha}+\pi^{\dot{\alpha}} \partial_{\dot{\alpha}}\right) \\
V(R) & =\lambda^{\alpha} \partial_{\alpha}-\pi^{\dot{\alpha}} \partial_{\dot{\alpha}} .
\end{aligned}
$$

The vector fields generating linearly realised $Q$-supersymmetry are

$$
\begin{aligned}
& V(Q)_{\alpha}=\pi^{\dot{\alpha}} \partial_{\alpha \dot{\alpha}}+y \partial_{\alpha} \\
& V(Q)_{\dot{\alpha}}=\lambda^{\alpha} \partial_{\alpha \dot{\alpha}}-y \partial_{\dot{\alpha}}
\end{aligned}
$$

while those generating linearly realised $S$-supersymmetry are

$$
\begin{aligned}
V(S)^{\alpha} & =x^{\alpha \dot{\alpha}} \partial_{\dot{\alpha}}+\lambda^{\alpha} \partial_{y}, \\
V(S)^{\dot{\alpha}} & =x^{\alpha \dot{\alpha}} \partial_{\alpha}-\pi^{\dot{\alpha}} \partial_{y} .
\end{aligned}
$$

The remaining supersymmetry transformations are the non-linearly realised $S$-supersymmetries generated by

$$
\begin{aligned}
V(S)^{\dot{\alpha}} & =x^{\beta \dot{\alpha}} \pi^{\dot{\beta}} \partial_{\beta \dot{\beta}}+x^{\beta \dot{\alpha}} y \partial_{\beta}-\pi^{\dot{\alpha}} \pi^{\dot{\beta}} \partial_{\dot{\beta}}-\pi^{\dot{\alpha}} y \partial_{y}, \\
V(S)^{\alpha} & =-\lambda^{\beta} x^{\alpha \dot{\beta}} \partial_{\beta \dot{\beta}}-\lambda^{\beta} \lambda^{\alpha} \partial_{\beta}+y x^{\alpha \dot{\beta}} \partial_{\dot{\beta}}+y \lambda^{\alpha} \partial_{y} .
\end{aligned}
$$


Finally, we have conformal boosts $(K)$ and internal conformal boosts $\left(K^{\prime}\right)$ generated by

$$
\begin{aligned}
V(K)^{\alpha \dot{\alpha}} & =x^{\beta \dot{\alpha}} x^{\alpha \dot{\beta}} \partial_{\beta \dot{\beta}}+x^{\beta \dot{\alpha}} \lambda^{\alpha} \partial_{\beta}+\pi^{\dot{\alpha}} x^{\alpha \dot{\beta}} \partial_{\dot{\beta}}+\pi^{\dot{\alpha}} \lambda^{\alpha} \partial_{y}, \\
V\left(K^{\prime}\right) & =\lambda^{\beta} \pi^{\dot{\beta}} \partial_{\beta \dot{\beta}}+\lambda^{\beta} y \partial_{\beta}+y \pi^{\dot{\beta}} \partial_{\dot{\beta}}+y^{2} \partial_{y} .
\end{aligned}
$$

The function $\Delta$ is non-zero in the following cases:

$$
\begin{aligned}
\Delta(D) & =1, \\
\Delta\left(D^{\prime}\right) & =-\frac{1}{2}, \\
\Delta(S)^{\dot{\alpha}} & =\pi^{\dot{\alpha}}, \\
\Delta(S)^{\alpha} & =-\lambda^{\alpha}, \\
\Delta(K)^{\alpha \dot{\alpha}} & =x^{\alpha \dot{\alpha}}, \\
\Delta\left(K^{\prime}\right) & =-y .
\end{aligned}
$$

In these lists for $V$ and $\Delta$ we have omitted the parameters which of course have the opposite indices to those displayed. The parameters can easily be restored, but this should be done from the left in order to get the right signs.

\subsection{Analytic fields}

We recall that a holomorphic tensor field of charge $p$ on $\mathbb{C} P^{1}$ is given by two local functions $a, a^{\prime}$ on $U, U^{\prime}$ respectively, such that, in the overlap

$$
a(y)=y^{p} a^{\prime}\left(y^{\prime}\right)
$$

Expanding both sides in power series in their respective variables, equating powers of $y\left(=\frac{1}{y^{\prime}}\right)$ and demanding the absence of poles we find

$$
a(y)=\sum_{n=0}^{n=p} a_{n} y^{n}
$$

and similarly for $a^{\prime}\left(y^{\prime}\right)$. The two expansions are related by

$$
a_{n}=a_{p-n}^{\prime}
$$

Hence the space of tensor fields of charge $p$ is a finite-dimensional space with dimension $p+1$ which can be identified with the space of $p$ th rank totally symmetric tensors under $S L(2)$.

In a similar fashion we define an analytic superfield of charge $p$ on $M_{A}$ to be specified by two local holomorphic functions $\phi(x, \lambda, \pi, y)$ and $\phi^{\prime}\left(x^{\prime}, \lambda^{\prime}, \pi^{\prime}, y^{\prime}\right)$ defined on the two standard coordinate patches $U, U^{\prime}$ respectively, such that, in the overlap

$$
\phi(x, \lambda, \pi, y)=y^{p} \phi^{\prime}\left(x^{\prime}, \lambda^{\prime}, \pi^{\prime}, y^{\prime}\right)
$$


If we now expand both sides in the odd variables and in $y$ or $y^{\prime}$, we obtain restrictions on the component functions. For example, the zeroth order term in $\lambda \pi$ is an $x$-dependent charge $p$

holomorphic tensor on $\mathbb{C} P^{1}$, the component of $\lambda$ behaves like a tensor of charge $p-1$ and so on. In addition, since the relation between $x$ and $x^{\prime}$ involves a shift, spacetime derivatives will appear in the constraints.

The basic superfield we shall consider is the hypermultiplet. In this language this multiplet is represented by an analytic superfield $\phi$ of charge 1 . Using the above method one can easily seen that it has only a short expansion:

$$
\phi(x, \lambda, \pi, y)=\varphi(x, y)+\lambda^{\alpha} \psi_{\alpha}(x)+\pi^{\dot{\alpha}} \chi_{\dot{\alpha}}(x)+\lambda^{\alpha} \pi^{\dot{\alpha}} \check{\varphi}_{\alpha \dot{\alpha}}(x)
$$

Furthermore

$$
\varphi(x, y)=\varphi_{o}(x)+y \varphi_{1}(x)
$$

and similarly for $\varphi^{\prime}\left(x^{\prime}, y^{\prime}\right)$, with

$$
\varphi_{o}=\varphi_{1}^{\prime} ; \quad \varphi_{1}=\varphi_{o}^{\prime}
$$

We also find

$$
\check{\varphi}_{\alpha \dot{\alpha}}=-\partial_{\alpha \dot{\alpha}} \varphi_{1} ; \quad \check{\varphi}_{\alpha \dot{\alpha}}^{\prime}=\partial_{\alpha \dot{\alpha}} \varphi_{o} .
$$

In addition, the fields $\varphi_{o}, \varphi_{1}, \psi, \chi$ must all satisfy their equations of motion

$$
\begin{aligned}
\square \varphi_{o}=\square \varphi_{1} & =0, \\
\partial^{\dot{\alpha} \alpha} \psi_{\alpha} & =0, \\
\partial^{\alpha \dot{\alpha}} \chi_{\dot{\alpha}} & =0 .
\end{aligned}
$$

This is the usual hypermultiplet with two complex scalar fields and two complex Weyl fermions, all of which are physical and on-shell. In the interacting theory, the on-shell hypermultiplet will be covariantly analytic, but gauge-invariant products of hypermultiplets will be analytic tensor fields of the type we have just described with charges $2,3, \ldots$ For example, a field of charge two contains an independent vector field $v_{\alpha \dot{\alpha}}$, which is conserved, but there are no equations of motion. In other words a charge two field is a linear multiplet.

\subsection{Correlation functions and Ward identities}

In this section we consider the superconformal Ward identities for four-point correlation functions for analytic operators with charges $p_{1}, p_{2}, p_{3}, p_{4}$. We shall denote such correlators by $<p_{1} p_{2} p_{3} p_{4}>$. Note, however, that the operators are not assumed to be the same even if they have the same charges, in particular, we shall not impose any symmetry requirements on such correlators.

If we assume that analyticity holds in the quantum theory, the superconformal Ward identity for such a correlator reads

$$
\sum_{i=1}^{4}\left(V_{i}+p_{i} \Delta_{i}\right)<p_{1} p_{2} p_{3} p_{4}>=0
$$


The assumption of analyticity is tantamount to using the field equations of the underlying hypermultiplet at operator level. This should be reasonable provided that we keep the points separated. In the case of charge two operators, which is the main focus of this paper, the $\mathrm{H}-$ analyticity condition implies, as we noted above, that the superfield describes a linear multiplet with a conserved spacetime current. Analyticity can be examined directly in perturbation theory using the harmonic superspace formalism and has been verified in all the examples that have been looked at so far.

We shall consider correlators of the above type which have non-vanishing leading terms. Further, we will specialise to correlators with four equal charges $p$. In this case we can write

$$
<p p p p>=\left(g_{12}\right)^{p}\left(g_{34}\right)^{p} F
$$

where $g_{i j}$ is the free two-point function for charge one operators at points $i$ and $j$,

$$
g_{i j}=\operatorname{sdet} X_{i j}^{-1}=\frac{\hat{y}_{i j}}{x_{i j}^{2}}
$$

and $F$ is an arbitrary function of invariants. Here $X_{i j}=X_{i}-X_{j}$ denotes the coordinate difference matrix for points $i$ and $j$ and

$$
\hat{y}_{i j}=y_{i j}-\pi_{i j} x_{i j}^{-1} \lambda_{i j}
$$

with the index convention that $x^{-1}$ has a pair of subscript indices $\dot{\alpha} \alpha$.

It is straightforward to check that $g_{i j}$ satisfies the equation

$$
\left(V_{i}+V_{j}+\Delta_{i}+\Delta_{j}\right) g_{i j}=0
$$

so that the Ward identity for $<p p p p>$ will indeed be satisfied for any invariant $F$.

It is not difficult to see that there must be more $N=2$ analytic superconformal invariants than there are spacetime conformal invariants for four points. If we expand $F$ in the odd variables $\lambda$ and $\pi$ the leading term must be invariant under spacetime conformal transformations and also under conformal $(S L(2))$ transformations of $\mathbb{C} P^{1}$. At four points there are two independent spacetime $(x)$ cross-ratios and one independent internal $(y)$ cross-ratio, so that there should be at least three independent analytic superconformal invariants at four points. In fact, there are no more. Any additional independent invariant would have to be nilpotent, but one can easily show that there are no such invariants at four points. The reason is essentially due to counting; there are $4 \times 4=16$ odd coordinates which is precisely equal to the number of supersymmetries in $S L(4 \mid 2)$. On a putative nilpotent invariant these supersymmetries behave essentially like translational symmetr! ies so that any possible leading term must vanish. In more detail, suppose that $F$ is a nilpotent four-point invariant. It can be written $F_{o}+\ldots$ where $F_{o}$ is the term with the lowest power of $\lambda \pi$. By examining the supersymmetry transformations directly one can see that the first term in the variation of $F$ involves only $F_{o}$. Furthermore, again by looking at each transformation in turn, one finds that setting this first term in the variation equal to zero (because $F$ is an invariant) leaves no possible solutions for separated points. Consequently one concludes that there can be no nilpotent invariants. This argument is presented in detail in [21].

It was shown in [6] that non-nilpotent analytic superspace superconformal invariants can be expressed in terms of superdeterminants and supertraces of the coordinate differences $X_{i j}$. For the case in hand a possible choice of three independent invariants is given as follows: we take two super cross-ratios 


$$
S=\frac{\operatorname{sdet} X_{14} \operatorname{sdet} X_{23}}{\operatorname{sdet} X_{12} \operatorname{sdet} X_{34}}, \quad T=\frac{\operatorname{sdet} X_{13} \operatorname{sdet} X_{24}}{\operatorname{sdet} X_{12} \operatorname{sdet} X_{34}}
$$

and one supertrace invariant

$$
U=\operatorname{str}\left(X_{12}^{-1} X_{23} X_{34}^{-1} X_{41}\right)
$$

The invariants $S$ and $T$ may be expressed in terms of the spacetime cross-ratios

$$
s=\frac{x_{14}^{2} x_{23}^{2}}{x_{12}^{2} x_{34}^{2}}, \quad t=\frac{x_{13}^{2} x_{24}^{2}}{x_{12}^{2} x_{34}^{2}}
$$

and the internal cross-ratio

$$
v=\frac{y_{14} y_{23}}{y_{12} y_{34}}
$$

in the form

$$
S=\frac{s}{\hat{v}}, \quad T=\frac{t}{\hat{w}} .
$$

Here $w=\frac{y_{13} y_{24}}{y_{12} y_{34}}=1+v$, and the hats on $v$ and $w$ are defined by hatting each of the $y$ variables in their definitions,

$$
\hat{v}=\frac{\hat{y}_{14} \hat{y}_{23}}{\hat{y}_{12} \hat{y}_{34}}, \quad \hat{w}=\frac{\hat{y}_{13} \hat{y}_{24}}{\hat{y}_{12} \hat{y}_{34}} .
$$

We may write

$$
\hat{w}=1+\hat{v}+\Delta w
$$

and express the third invariant $U$ in the form

$$
U=1-t+s+\hat{v}+\Delta U
$$

Both $\Delta W$ and $\Delta U$ are nilpotent quantities. The explicit expressions are as follows:

$$
\begin{aligned}
\Delta U= & \frac{1}{\hat{y}_{12} \hat{y}_{34}}\left(-\hat{y}_{12} \Pi_{2} x_{32}^{1} \Lambda_{2}+\hat{y}_{34} \Pi_{1} x_{23}^{4} \Lambda_{1}\right. \\
& +\hat{y}_{23}\left(\Pi_{1} x_{21}^{4} \Lambda_{1}-\Pi_{2} x_{34}^{1} \Lambda_{2}-\Pi_{1} \bar{x}_{21}^{4} \Lambda_{2}+\Pi_{2} \bar{x}_{34}^{1} \Lambda_{1}\right) \\
& \left.+\left(\Pi_{1} x_{23} \Lambda_{2}\right)\left(\Pi_{2} x_{23} \Lambda_{1}\right)\right)
\end{aligned}
$$

and 


$$
\begin{aligned}
\Delta w= & \frac{1}{\hat{y}_{12} \hat{y}_{34}}\left(-\hat{y}_{12} \Pi_{2} x_{32}^{4} \Lambda_{2}+\hat{y}_{34} \Pi_{1} x_{23}^{1} \Lambda_{1}\right. \\
& +\hat{y}_{23}\left(\Pi_{1}\left[x_{23}^{1}-x_{24}^{1}\right] \Lambda_{1}+\Pi_{2}\left[x_{31}^{4}-x_{32}^{4}\right] \Lambda_{2}+\Pi_{1} \bar{x}_{24}^{1} \Lambda_{2}-\Pi_{2} \bar{x}_{31}^{4} \Lambda_{1}\right) \\
& \left.-\left(\Pi_{1} x_{21}^{3} \Lambda_{1}\right)\left(\Pi_{2} x_{34}^{2} \Lambda_{2}\right)\right)
\end{aligned}
$$

where we have used the convenient shorthand

$$
\begin{aligned}
x_{i j}^{k} & =x_{i k} x_{j k}^{-1} x_{i j}, \\
\bar{x}_{i j}^{k} & =x_{i k} x_{j k}^{-1} x_{j l} \quad l \neq, i, j, k .
\end{aligned}
$$

The odd variables are defined by

$$
\begin{aligned}
& \Lambda_{1 \dot{\alpha}}=\left(x_{12}\right)_{\dot{\alpha} \alpha}^{-1} \lambda_{12}^{\alpha}-\left(x_{23}\right)_{\dot{\alpha} \alpha}^{-1} \lambda_{23}^{\alpha} \\
& \Lambda_{2 \dot{\alpha}}=\left(x_{23}\right)_{\dot{\alpha} \alpha}^{-1} \lambda_{23}^{\alpha}-\left(x_{34}\right)_{\dot{\alpha} \alpha}^{-1} \lambda_{34}^{\alpha}
\end{aligned}
$$

and, similarly,

$$
\begin{aligned}
\Pi_{1 \alpha} & =\pi_{12}^{\dot{\alpha}}\left(x_{12}\right)_{\dot{\alpha} \alpha}^{-1}-\pi_{23}^{\dot{\alpha}}\left(x_{23}\right)_{\dot{\alpha} \alpha}^{-1} \\
\Pi_{2 \alpha} & =\pi_{23}^{\dot{\alpha}}\left(x_{23}\right)_{\dot{\alpha} \alpha}^{-1}-\pi_{34}^{\dot{\alpha}}\left(x_{34}\right)_{\dot{\alpha} \alpha}^{-1}
\end{aligned}
$$

Now the crucial point is the following: each of the operators in the correlator can be expanded as a polynomial in $y$, so that the correlator is manifestly analytic in the $y$ variables. On the other hand, each of the invariants depend on the $y$ 's in a rational manner so that one might expect the absence of singularities to impose further constraints on $F$. These constraints will clearly depend on the charges involved. For the correlator $<p p p p>$ the lowest term in $F$ must be of the form

$$
\left.F\right|_{\lambda=\pi=0}=a_{0}+a_{1} v+\ldots a_{p+1} v^{p+1}
$$

where each of the $a$ 's depends on the cross-ratios $s, t$. The question then arises whether there are further constraints on the coefficient functions at higher orders. It is clear that the lower the charge the more constrained $F$ must be. For charge one, there are no gauge-invariant operators, so the simplest interesting case to examine is charge 2 to which we turn in the next section.

\subsection{Analyticity analysis}

In this section we analyse the constraints imposed by H-analyticity on the four-point function of four charge two operators (not necessarily the same). It can be written

$$
<2222>=\frac{\hat{y}_{12}^{2} \hat{y}_{34}^{2}}{x_{12}^{4} x_{34}^{4}} F(S, T, U)
$$


The invariants $S, T, U$ are convenient from some points of view - it is easy to see that they are invariants, and they have concise explicit forms. Requiring $\mathrm{H}$-analyticity implies that $F$ should have no singularities in $\left(\hat{y}_{13}, \hat{y}_{14}, \hat{y}_{23}, \hat{y}_{24}\right)$ and that it can have poles up to order two in $\left(y_{12}, y_{34}\right)$ (if we had charge $p$ operators this would become order $p$ ). Since $\left(\hat{y}_{12} \hat{y}_{34}\right)$ occurs as a denominator in $S^{-1}, T^{-1}$ and $U$ the regularity of the correlator will lead to constraints for $F$.

Note that regularity in the hatted variables is equivalent to regularity in the unhatted ones: if we demand that the whole correlator be a polynomial in the hatted $y$ 's and write, for example, $\hat{y}_{12}=y_{12}+\delta_{12}$, then a Taylor expansion will produce a polynomial of the same degree in the unhatted $y$ 's, because the $\delta$ 's are non-singular and $y$-independent.

Clearly, H-analyticity should hold at each order in the odd variables separately. We shall carry out the expansion in two steps: we first expand in the nilpotent quantities $\Delta U, \Delta w$ and then express the latter in terms of products of spinors. We will here refer to the order in $\Delta U,, \Delta w$ as "level" in order to distinguish the first from the second step. The main technical problem one faces in this approach is to compute which of the various products of the form $(\Delta u)^{p}(\Delta w)^{q}$ are independent for each fixed value of $p+q$, i.e. at a given "level".

From the point of view of expanding in odd variables it is helpful to think about an equivalent set of invariants, $S^{\prime}, T^{\prime}, V$, which are defined to have leading terms $s, t$ and $v$ respectively. These invariants are expressed in terms of $S, T, U$ by

$$
S^{\prime}=S V, \quad T^{\prime}=T(1+V), \quad V=\frac{T+U-1}{1+S-T}
$$

Since we have

$$
\begin{aligned}
S^{\prime} & =s+\ldots \\
T^{\prime} & =t+\ldots \\
V & =v+\ldots
\end{aligned}
$$

it follows from (51) that we may write $F$ in the form

$$
F=a_{1}\left(S^{\prime}, T^{\prime}\right)+a_{2}\left(S^{\prime}, T^{\prime}\right) V+a_{3}\left(S^{\prime}, T^{\prime}\right) V^{2} .
$$

This expression clearly meets the lowest level requirements of analyticity for charge 2 and shows that the dependence on the third invariant $V$ is thereby fixed. The objective now is to Taylor expand $\mathrm{F}$ about $S^{\prime}=s, T^{\prime}=t$ and $V=\hat{v}$. However, since the original set of invariants is easier to evaluate explicitly, we shall convert the Taylor expansion back into these variables as we go. In this way we arrive at a power series in the nilpotent quantities $\Delta U$ and $\Delta w$. We will then express both of these and the coefficients that accompany them in terms of the set of coordinates $\left(x_{12}, x_{23}, x_{34}, \hat{y}_{12}, \hat{y}_{23}, \hat{y}_{34}, \Lambda_{1}, \Lambda_{2}, \Pi_{1}, \Pi_{2}\right)$. These variables are convenient in the sense that they are invariant under translational $Q$-supersymmetry and linear $S$-supersymmetry, and in addition they involve no $y$-singularities. Furthermore, as noted above, the difference between $y$ and $\hat{y}$ is non-singular so it is permissible to study analyticity in the latter rather than the former.

The Taylor expansion of $F$ is then

$$
\begin{gathered}
=F_{o}+\Delta U\left(\partial_{U} F\right)+\Delta w\left(-\frac{t}{\bar{w}^{2}} \partial_{T} F\right) \\
\frac{1}{2} \Delta U^{2}\left(\partial_{U}^{2} F\right)+\Delta U \Delta w\left(-\frac{t}{\bar{w}^{2}} \partial_{U} \partial_{T} F\right)+\frac{1}{2} \Delta w^{2}\left(\frac{t^{2}}{\bar{w}^{4}} \partial_{T}^{2} F+2 \frac{t}{\bar{w}^{3}} \partial_{T} F\right)+\ldots
\end{gathered}
$$

where $F_{o}$ is $F\left(S^{\prime}, T^{\prime}, V\right)$ evaluated at $(s, t, \hat{v})$. Clearly 


$$
F_{o}=a_{1}(s, t)+a_{2}(s, t) \hat{v}+a_{3}(s, t) \hat{v}^{2} .
$$

In the new variables the partial derivatives, evaluated at $\left(S^{\prime}, T^{\prime}, \hat{v}\right)$, are

$$
\begin{aligned}
\partial_{U} & =\frac{1}{R} D \\
\partial_{T} & =\frac{(1+\hat{v})}{R}\left(D+R \partial_{t}\right)
\end{aligned}
$$

with

$$
\begin{aligned}
R & =s+\hat{v}-\frac{\hat{v} t}{1+\hat{v}} \\
D & =s \partial_{s}+\hat{v} \partial_{\hat{v}}+\frac{\hat{v} t}{1+\hat{v}} \partial_{t}
\end{aligned}
$$

Here $v$ has been replaced by $\hat{v}$ because we wish to use the Taylor expansion about the point $(s, t, \hat{v})$. We will almost always multiply $R$ and $D$ by $\bar{w}=1+\hat{v}$ in order to avoid the singularity in their denominators. Note that $D R=R$.

We shall refer to the expressions in round brackets multiplying a certain power $\Delta U^{p} \Delta w^{q}$ in (57) as "component functions". The expansion ends at fourth order, because $\Delta U, \Delta w$ are $R$ symmetric and are therefore power series in $(\Pi \Lambda)$. Since the spinors are two-component objects and since there are two $\Pi$ 's and two $\Lambda$ 's it follows that the highest possible power is $(\Pi \Lambda)^{4}$.

Let us investigate the linear level in the Taylor expansion. We ask whether singularities in $\Delta U$ and $\Delta W$ can conspire to cancel or whether the latter are independent objects. The component functions depend on $s, t, \hat{v}$, which yields a linear dependence problem with coefficients in the ring of functions of $s, t, \hat{v}$.

The functional form of these coefficients can be made much more explicit. Given the form of $F(58)$, by commuting all $R$ 's to the left and all $\partial_{t}$ 's to the right we can show that the general component functions at $k$-th level have the form

$$
\frac{1}{(\bar{w}(\bar{w} R))^{k}} \sum_{n=0}^{2+2 k} c_{n}(s, t) \hat{v}^{n} .
$$

The extra factors $\bar{w}$ in the denominator are introduced by the coefficients of the $\partial_{T}$ derivatives and obviously factor out in the pure $\left(\partial_{U}\right)^{k}$ component functions. A direct computer calculation shows them to cancel in the other components, too. Without enhanced factorisation abilities this point is hard to show and therefore we keep the $\bar{w}$ 's in the scheme. Incidentally, by introducing $\tau=1 / T$ the component functions can be more easily computed: They are simply

$$
\frac{1}{t^{n}} \partial_{\tau}^{n} \partial_{U}^{m} F
$$

We now examine the first order independence problem in detail. We work to lowest order, so the hat on $v$ is left out in the following and $R_{0}$ denotes the body of $R$. For charge two operators analyticity requires that there be functions of $(s, t)$ for $\Delta U$ and $\Delta w$ such that 


$$
\Delta U \frac{1}{w\left(w R_{0}\right)}\left(\sum_{n=0}^{4} c_{0 n}(s, t) v^{n}\right)+\Delta w \frac{1}{w\left(w R_{0}\right)}\left(\sum_{n=0}^{4} c_{1 n}(s, t) v^{n}\right)=O\left(\frac{1}{\left(\hat{y}_{12} \hat{y}_{34}\right)^{2}}\right)
$$

with $w=1+v$.

Spinors occur in $\Delta U, \Delta w$ in combinations of the form $\left(\Pi x x^{-1} x \Lambda\right)$. Minkowski space is fourdimensional; a basis consists of four independent elements. In order to express the various $x$-triples in a given basis, we use

$$
x_{i} x_{j}^{\dagger}+x_{j} x_{i}^{\dagger}=2\left(x_{i} \cdot x_{j}\right) \delta
$$

to commute $x_{12}$ left of $x_{23}$ left of $x_{34} . \quad\left(\left(x_{i} \cdot x_{j}\right)\right.$ means the dot product of the associated four-vectors.) In this way the spinors are seen to be contracted on elements of the basis $\left\{x_{12}, x_{23}, x_{34}, x_{12} x_{23}^{\dagger} x_{34}\right\}$.

There are two $\Pi_{i}$ and two $\Lambda_{i}$ so that we get a total of sixteen independent structures at first order in $(\Pi \Lambda)$. A dependence relation between $\Delta U$ and $\Delta w$ is a linear combination with scalar coefficients which is of a certain order in $\hat{y}$-singularities in all sixteen components separately. We restrict the analysis to the $\left(\Pi_{1} \Lambda_{2}\right)$ part of the odd expansion:

$$
\begin{array}{cc}
\left.\Delta U\right|_{\Pi_{1} \Lambda_{2}}= & -\frac{\hat{y}_{23}}{\hat{y}_{12} \hat{y}_{34}} \Pi_{1}\left(x_{34}^{2} x_{12}+x_{14}^{2} x_{23}+x_{12}^{2} x_{34}+x_{12} x_{23}^{\dagger} x_{34}\right) \Lambda_{2}, \\
\left.\Delta w\right|_{\Pi_{1} \Lambda_{2}}= & -\frac{\hat{y}_{23}}{\hat{y}_{12} \hat{y}_{34}} \Pi_{1}\left(x_{34}^{2} x_{12}+x_{12}^{2} x_{34}+x_{12} x_{23}^{\dagger} x_{34}\right) \Lambda_{2}
\end{array}
$$

which we write in short as

$$
\begin{aligned}
\left.\Delta U\right|_{\Pi_{1} \Lambda_{2}} & =-\frac{\hat{y}_{23}}{\hat{y}_{12} \hat{y}_{34}} \Pi_{1} X_{U} \Lambda_{2}, \\
\left.\Delta w\right|_{\Pi_{1} \Lambda_{2}} & =-\frac{\hat{y}_{23}}{\hat{y}_{12} \hat{y}_{34}} \Pi_{1} X_{w} \Lambda_{2} .
\end{aligned}
$$

On Minkowski space we change basis to $\left\{X_{U}, X_{w}, x_{12}, x_{34}\right\}$ upon which the equation (63) breaks into two separate parts. We can omit the spinors and the $x$-vectors from the discussion as they have to be equal on both sides of the equations. This leads to the scalar equation

$$
-\frac{\hat{y}_{23}}{\hat{y}_{12} \hat{y}_{34}}\left(\sum_{i=0}^{4} c_{n}(s, t) v^{n}=(1+v)\left(s+v(1+s-t)+v^{2}\right) O\left(\frac{1}{\left(\hat{y}_{12} \hat{y}_{34}\right)^{2}}\right)\right.
$$

for both the $\Delta U$ and $\Delta W$ parts.

Instead of the variables $\left\{\hat{y}_{12}, \hat{y}_{23}, \hat{y}_{34}\right\}$ we may choose the set $\left\{v, Y_{p}=\hat{y}_{23} /\left(\hat{y}_{12} \hat{y}_{34}\right), \hat{y}_{23}\right\}$. The Jacobian of the transform is

$$
J=\frac{\hat{y}_{23}^{2}}{\left(\hat{y}_{12} \hat{y}_{34}\right)^{3}}\left(\hat{y}_{12}-\hat{y}_{34}\right)
$$

and is regular at a generic point. Given this choice, the L.H.S. of (69) is independent of $\hat{y}_{23}$ and factors out a single power of $Y_{p}$. We can conclude that the same is true for the R.H.S. and hence the as yet unspecified term is a function of $s, t, v$ of maximum order $1 /\left(\hat{y}_{12} \hat{y}_{34}\right)$. It must be a polynomial of degree 1 in $v$. 
It follows that the ansatz polynomial on the L.H.S. factors in the same way and $w\left(w R_{0}\right)$ cancels from the equation. The only solution of the regularity problem at first order is therefore the trivial one:

$$
\Delta U\left(\sum_{n=0}^{1} g_{n}(s, t) v^{n}\right)+\Delta w\left(\sum_{n=0}^{1} h_{n}(s, t) v^{n}\right)=O\left(\frac{1}{\left(\hat{y}_{12} \hat{y}_{34}\right)^{2}}\right)
$$

This is a general solution because $\Delta U$ and $\Delta w$ are not more singular than $1 /\left(\hat{y}_{12} \hat{y}_{34}\right)$ in any of the components w.r.t. the sixteen independent structures at first order. By inspection, the higher order terms arising from the second order in $\Delta U, \Delta W$ and from the soul of $\hat{v}$ will also be regular.

Let us state the result again: The explicit forms for the level one component functions in the Taylor expansion (57) must have the dependence on $\hat{v}$ indicated by the above equation. Hence

$$
\frac{D F_{o}}{R}=g_{1}+\hat{v} g_{2}
$$

and

$$
\frac{\left(D+R \partial_{t}\right) F_{o}}{\bar{w} R}=h_{1}+\hat{v} h_{2} .
$$

We ought to stress that the proof makes use of certain regularity assumptions. Throughout the calculation we have assumed the $x$-scalars and in particular $s$ and $t$ to be regular. Hence none of the four points can be light-like separated in Minkowski space. This can possibly be relaxed. A necessary assumption is certainly that the vector basis is non-degenerate, so in particular the points do not coincide. For the $\hat{y}$-coordinates we must also demand that the points are distinct. For the Jacobian (70) to be non-vanishing we additionally need $\hat{y}_{12} \neq \hat{y}_{34}$.

If $\hat{y}_{34}=\alpha \hat{y}_{12}$ we can change from $\left\{\hat{y}_{12}, \hat{y}_{23}\right\}$ to $\left\{\hat{y}_{23} /\left(\alpha \hat{y}_{12}^{2}\right), v\right\}$. In this case (69) still has the same consequences: The prefactor must occur on both sides and drops out. The unknown part on the R.H.S. is a function of $s, t, v$ as before. The Jacobian of the transformation is

$$
J=\frac{\hat{y}_{23}}{\alpha^{2} \hat{y}_{12}^{5}}\left((1+\alpha) \hat{y}_{12}+2 \hat{y}_{23}\right)
$$

and hence is regular if the points do not coincide and $\hat{y}_{12}$ is not proportional to $\hat{y}_{23}$, so if there is more than one difference variable. The argument holds in fact as long as two of the $\hat{y}_{i j}$ are independent. Otherwise $v$ is a constant and there is nothing to discuss.

The generalisation of the independence problem (63) to the higher levels like $\Delta U^{2}, \Delta U \Delta w, \ldots$ is obvious. We have investigated this in the same manner to lowest order and we find that the spinor combinations are not independent. Additionally, the simple argument does not apply, which allowed us to ignore higher orders stemming from the lower level terms in the Taylor expansion (57). These terms define a non-trivial right hand side in the equivalent of (63) in the levels above. Due to linearity it suffices to find one special solution to this inhomogeneous problem which is to be added to the general solution of the homogeneous one.

To analyse equation (72) we begin by multiplying it by $(1+\hat{v}) R$ which gives a polynomial equation in $\hat{v}$. This allows us to express the unknown functions $g_{1}, g_{2}$ in terms of $a_{1}, a_{2}, a_{3}$,

$$
g_{1}=a_{1 s}, \quad g_{2}=a_{1 s}+a_{1 t}-a_{2 t}+a_{3 t}
$$


where the literal subscripts denote partial derivatives. This leaves two first-order partial differential equations for $a_{1}, a_{2}, a_{3}$,

$$
\begin{aligned}
(t-s-1) a_{1 s}+(t-s) a_{1 t}+s\left(a_{2 s}+a_{2 t}\right)+a_{2}-s a_{3 t} & =0 \\
-a_{1 s}-a_{1 t}+a_{2 t}+s a_{3 s}+(t-1) a_{3 t}+2 a_{3} & =0 .
\end{aligned}
$$

If we make the change of variables

$$
\begin{aligned}
& a_{1}=\alpha+\gamma+s a_{3} \\
& a_{2}=\gamma+(s-t+1) a_{3}
\end{aligned}
$$

with $a_{3}$ unchanged, we find that $a_{3}$ drops out of the above equations altogether - it is completely undetermined. The equations are satisfied if

$$
\begin{aligned}
\alpha_{s}+\alpha_{t}+\gamma_{s} & =0 \\
t \gamma_{t}+\gamma+(1-s) \alpha_{t}-s \alpha_{s} & =0
\end{aligned}
$$

This pair of first-order coupled differential equations can be equivalently rewritten as a set of second-order independent ones:

$$
\begin{aligned}
s \alpha_{s s}+t \alpha_{t t}+(s+t-1) \alpha_{s t}+2\left(\alpha_{s}+\alpha_{t}\right) & =0 \\
s \gamma_{s s}+t \gamma_{t t}+(s+t-1) \gamma_{s t}+2\left(\gamma_{s}+\gamma_{t}\right) & =0 .
\end{aligned}
$$

Note that in the correlator there is still one arbitrary coefficient function $\left(a_{3}(s, t)\right.$ for this choice of variables) in addition to the solutions to these equations.

When carrying out this type of analysis for the higher level regularity problems, we find that the dependence relations between $\Delta U^{k}$ etc. introduce so many unknowns into the equations that no new constraints are found. We have done these calculations for operator weight one through three with the result that the only constraints arise from the first level.

\section{$3 \quad S U(2)$-covariant approach}

In this section we explain how the four-point function results obtained in the first part of this paper can be found in an independent way in a harmonic superspace formulation which maintains the explicit $S U(2)$ covariance. The technique is quite different, the main point being that here we shall keep Q supersymmetry (including its $S U(2)$ automorphism) manifest at each step. However, S supersymmetry, as well as harmonic analyticity will have to be checked level by level in the $\theta$ expansion of the four-point function. The advantage of this approach is the possibility to reformulate the $\mathrm{H}$-analyticity condition in an equivalent way which will allow us to essentially eliminate the dependence on the G-analytic Grassmann variables. After this the constraints at levels 1 and 2 become sufficiently easy to work out. Moreover, it becomes obvious that there are no constraints beyond level 2 . 


\section{1 $S U(2)$ harmonics and Grassmann analyticity}

As discussed in the previous section $N=2$ harmonic superspace is the product of super Minkowski space (coordinates $\left(x^{\mu} \sim x^{\alpha \dot{\alpha}}, \theta_{i}^{\alpha}, \bar{\theta}^{\dot{\alpha} i}\right)$ ) and the two-sphere. However, in this section, rather than using explicit coordinates $(y, \bar{y})$ for the sphere, we shall use an alternative approach first proposed in [18] in which the sphere is described by harmonic variables $u_{i}^{ \pm}$defined as the two columns of an $S U(2)$ matrix; the index $i$ transforms under the (left) $S U(2)$ and \pm under an independent (right) $U(1)$ group. The components of $u$ have the defining properties:

$$
\left(\begin{array}{cc}
u_{1}^{+} & u_{1}^{-} \\
u_{2}^{+} & u_{2}^{-}
\end{array}\right) \in S U(2) \quad \Rightarrow \quad u_{i}^{-}=\left(u^{+i}\right)^{*}, \quad u^{+i} u_{i}^{-}=1
$$

where the $S U(2)$ indices are raised and lowered in the following way, $f^{i}=\epsilon^{i j} f_{j}, f_{i}=\epsilon_{i j} f^{j}$ with $\epsilon_{i j}$ defined by $\epsilon_{12}=-\epsilon^{12}=1$.

The harmonic functions $f^{q}\left(u_{i}^{ \pm}\right)$are defined as singlets of the left $S U(2)$ but they are homogeneous of degree $q$ under the right $U(1)$, i.e. carry a charge $q$. Effectively, such functions live on the coset $S U(2) / U(1) \sim S^{2}$ and are assumed to have a harmonic expansion on the sphere. A powerful feature of the coordinateless parametrisation of $S^{2}$ in terms of harmonics $u_{i}^{ \pm}$is the possibility to write down such expansions in a manifestly $S U(2)$ covariant way, e.g., for $q \geq 0$ :

$$
f^{q}(u)=\sum_{n=0}^{\infty} f^{\left(i_{1} \ldots i_{n+q} j_{1} \ldots j_{n}\right)} u_{i_{1}}^{+} \ldots u_{i_{n+q}}^{+} u_{j_{1}}^{-} \ldots u_{j_{n}}^{-} .
$$

The coefficients in this expansion are totally symmetric multispinors, i.e. irreps of $S U(2)$ of isospins $q / 2+n, n=0,1, \ldots$ Thus, using harmonic variables allows one to deal with $U(1)$ covariant objects without loosing the $S U(2)$ symmetry.

With the aid of $u$ we can define Grassmann analyticity in an $S U(2)$-covariant way. We split the Grassmann variables $\theta_{i}^{\alpha}, \bar{\theta}^{\dot{\alpha} i}$ into two $U(1)$ projections,

$$
\theta^{ \pm \alpha}=u_{i}^{ \pm} \theta^{i \alpha}, \quad \bar{\theta}^{ \pm \dot{\alpha}}=u_{i}^{ \pm} \bar{\theta}^{i \dot{\alpha}}
$$

still maintaining the $S U(2)$ invariance. We then define a G-analytic function $\phi$ on harmonic superspace to be one which satisfies

$$
D_{\alpha}^{+} \phi=\bar{D}_{\dot{\alpha}}^{+} \phi=0
$$

where the $U(1)$ projections of the supercovariant derivatives are defined in a similar way. These constraints are solved by

$$
\phi=\phi\left(x_{A}, \theta^{+\alpha}, \bar{\theta}^{=\dot{\alpha}}\right)
$$

where

$$
x_{A}^{\alpha \dot{\alpha}}=x^{\alpha \dot{\alpha}}-4 i \theta^{(i \alpha} \bar{\theta}^{j) \dot{\alpha}} u_{i}^{+} u_{j}^{-}
$$

with $x^{\alpha \dot{\alpha}}=x^{\mu} \sigma_{\mu}^{\alpha \dot{\alpha}}$. Clearly G-analyticity is a Q-supersymmetric notion because it involves the supercovariant derivatives.

As explained in the previous section, complexified analytic superspace can be defined as a coset space of the complex superconformal group. Although this is not possible in real spacetime 
we can nevertheless define a representation of the Lie superalgebra of $S U(2,2 \mid 2)$ on $G$-analytic fields. For all practical purposes this amounts to taking infinitesimal $S U(2)$ transformations (parameters $\lambda^{j k}$ ) to have the form $u_{i}^{ \pm}$:

$$
\delta u_{i}^{+}=\left(\lambda^{j k} u_{j}^{+} u_{k}^{+}\right) u_{i}^{-}, \quad \delta u_{i}^{-}=0 .
$$

More generally, it is sufficient to treat the transformations of the harmonic variables $u_{i}^{ \pm}$with

generators $K, D, R, S, I$ as active ones. For instance, a harmonic function $f^{(p)}(u)$ of weight $p$ will transform as follows:

$$
\begin{aligned}
\delta f^{(p)}(u) & =f^{\prime(p)}(u)-f^{(p)}(u) \\
& =-\left(\lambda^{i j} u_{i}^{+} u_{j}^{+}\right) u_{k}^{-} \frac{\partial}{\partial u_{k}^{+}} f^{(p)}(u)+p\left(\lambda^{i j} u_{i}^{-} u_{j}^{+}\right) f^{(p)}(u),
\end{aligned}
$$

so that the non-unitary transformation appears in the form of a derivative of a function of unitary harmonics.

Henceforth we shall be dealing exclusively with G-analytic fields so that we can replace $x_{A}$ by $x$ without loss of clarity. The actions of the supersymmetry transformations on the coordinates are given by

$$
\begin{aligned}
\delta_{Q} x^{\alpha \dot{\alpha}} & =-4 i u_{i}^{-}\left(\epsilon^{i \alpha} \bar{\theta}^{+\dot{\alpha}}+\theta^{+\alpha} \bar{\epsilon}^{i \dot{\alpha}}\right) \\
\delta_{Q} \theta^{+\alpha, \dot{\alpha}} & =u_{i}^{+} \epsilon^{i \alpha, \dot{\alpha}} \\
\delta_{Q} u_{i}^{ \pm} & =0
\end{aligned}
$$

and 25]

$$
\begin{aligned}
\delta_{S} x^{\alpha \dot{\alpha}} & =4 i\left(x^{\alpha \dot{\beta}} \bar{\theta}^{+\dot{\alpha}} \bar{\eta}_{\dot{\beta}}^{i}-x^{\dot{\alpha} \beta} \theta^{+\alpha} \eta_{\beta}^{i}\right) u_{i}^{-} \\
\delta_{S} \theta^{+\alpha} & =-2 i\left(\theta^{+}\right)^{2} \eta^{\alpha i} u_{i}^{-}+x^{\alpha \dot{\beta}} \bar{\eta}_{\dot{\beta}}^{i} u_{i}^{+} \\
\delta_{S} \theta^{-\alpha} & =4 i \eta_{\beta}^{i} \theta^{-\beta}\left(\theta^{-\alpha} u_{i}^{+}-\theta^{+\alpha} u_{i}^{-}\right)+\bar{\eta}_{\dot{\beta}}^{i}\left(x^{\alpha \dot{\beta}}+4 i \theta^{-\alpha} \bar{\theta}^{+\dot{\beta}}\right) u_{i}^{-} \\
\delta_{S} u_{i}^{+} & =\left[4 i\left(\theta^{+\alpha} \eta_{\alpha}^{i}+\bar{\eta}_{\dot{\alpha}}^{i} \bar{\theta}^{+\dot{\alpha}}\right) u_{i}^{+}\right] u_{i}^{-} \\
\delta_{S} u_{i}^{-} & =0
\end{aligned}
$$

$\left(\delta_{S} \bar{\theta}^{ \pm}\right.$are obtained by conjugation). From this one can compute the action of the rest of the superconformal algebra by commuting $\mathrm{Q}$ and $\mathrm{S}$ supersymmetry transformations.

\subsection{The hypermultiplet}

In this subsection we give the harmonic formulation of the hypermultiplet describing an $S U(2)$ doublet of scalars $f^{i}(x)$ and a pair of Weyl (complex) spinors $\psi_{\alpha}(x), \bar{\xi}^{\dot{\alpha}}(x)$ on shell. Off shell it can only exist with an infinite set of auxiliary fields [27]. Such a set is naturally provided by the G-analytic superfield of $U(1)$ charge +1 :

$$
q^{+}\left(x, \theta^{+}, \bar{\theta}^{+}, u\right)=F^{+}(x, u)+\theta^{+\alpha} \Psi_{\alpha}(x, u)+\bar{\theta}_{\dot{\alpha}}^{+} \bar{\Xi}^{\dot{\alpha}}(x, u)+\ldots+\left(\theta^{+}\right)^{2}\left(\bar{\theta}^{+}\right)^{2} P^{-3}(x, u) .
$$


The components of this $\theta^{+}$expansion are harmonic functions with infinite expansions on $S^{2}$ (see (81)):

$$
\begin{aligned}
F^{+}(x, u)= & f^{i}(x) u_{i}^{+}+f^{(i j k)}(x) u_{i}^{+} u_{j}^{+} u_{k}^{-}+\ldots \\
\Psi_{\alpha}(x, u)= & \left.\psi_{\alpha}(x)+\psi_{\alpha}^{(i j)}(x)\right) u_{i}^{+} u_{j}^{-}+\ldots \\
& \ldots \\
P^{-3}(x, u)= & p^{(i j k)}(x) u_{i}^{-} u_{j}^{-} u_{k}^{-}+\ldots
\end{aligned}
$$

The coefficients in these expansions are ordinary fields belonging to different $S U(2)$ representations. All of them, with the exception of the physical fields $f^{i}(x), \psi_{\alpha}(x), \bar{\xi}^{\dot{\alpha}}(x)$ are auxiliary, so they should vanish on shell. We need a way to write down a supersymmetric on-shell constraint on the G-analytic superfield $q^{+}\left(x, \theta^{+}, \bar{\theta}^{+}, u\right)$.

The key to this on-shell constraint is provided by the notion of harmonic (H-)analyticity. The harmonic coset $S U(2) / U(1)$ has two real (or one complex) dimensions, to which correspond the following harmonic derivatives:

$$
\begin{aligned}
& \partial^{++}=u_{i}^{+} \frac{\partial}{\partial u_{i}^{-}} \quad \Rightarrow \quad \partial^{++} u_{i}^{+}=0, \quad \partial^{++} u_{i}^{-}=u_{i}^{+}, \\
& \partial^{--}=u_{i}^{-} \frac{\partial}{\partial u_{i}^{+}} \quad \Rightarrow \quad \partial^{--} u_{i}^{+}=u_{i}^{-}, \partial^{--} u_{i}^{-}=0 .
\end{aligned}
$$

These are Cartan's covariant derivatives on the coset. In our context this simply means that they preserve the defining condition $u^{+i} u_{i}^{-}=1$. To them one may add the charge-counting operator

$$
\partial^{0}=u_{i}^{+} \frac{\partial}{\partial u_{i}^{+}}-u_{i}^{-} \frac{\partial}{\partial u_{i}^{-}} \Rightarrow \partial^{0} u_{i}^{ \pm}= \pm u_{i}^{ \pm}
$$

By definition all harmonic functions are eigenfunctions of $\partial^{0}, \partial^{0} f^{q}(u)=q f^{q}(u)$.

An important point is that the three covariant derivatives above form an $S U(2)$ algebra:

$$
\left[\partial^{++}, \partial^{--}\right]=\partial^{0}, \quad\left[\partial^{0}, \partial^{ \pm \pm}\right]= \pm 2 \partial^{ \pm \pm} .
$$

They can be regarded as the generators of right $S U(2)_{R}$ rotations acting on the indices \pm of the harmonics $u_{i}^{ \pm}$. Thus, $\partial^{++}$is the raising and $\partial^{--}$the lowering operator of $S U(2)_{R}$ (see (92)). This observation suggests the way to define short harmonic functions as highest weights of irreps of $S U(2)_{R}$. Thus, depending on the value of the $U(1)$ charge, the condition

$$
\partial^{++} f^{q}(u)=0 \Rightarrow\left\{\begin{array}{l}
f^{q}(u)=0, q<0 \\
f^{q}(u)=u_{i_{1}}^{+} \ldots u_{i_{q}}^{+} f^{\left(i_{1} \ldots i_{q}\right)}, q \geq 0
\end{array}\right.
$$

has either a trivial solution or defines an irrep of isospin $q / 2$. This property is a direct consequence of the general form (81) of the harmonic expansion on $S^{2}$ and of the action of $\partial^{++}$on the harmonics (92).

An alternative interpretation of the condition (95) is that of harmonic (H-)analyticity. Let us introduce stereographic coordinates on the sphere (see [28] for more detail): 


$$
\left(\begin{array}{ll}
u_{1}^{+} & u_{1}^{-} \\
u_{2}^{+} & u_{2}^{-}
\end{array}\right)=\frac{1}{\sqrt{1+y \bar{y}}}\left(\begin{array}{cc}
1 & -\bar{y} \\
y & 1
\end{array}\right)
$$

Our harmonic functions $f^{q}(u)$ are by definition eigenfunctions of the charge "operator" $\partial^{0}$ :

$$
\partial^{0} f^{q}(y, \bar{y})=q f^{q}(y, \bar{y})
$$

In this parametrisation the covariant derivative $\partial^{++}$becomes

$$
\partial^{++}=-(1+y \bar{y}) \frac{\partial}{\partial \bar{y}}-\frac{y}{2} \partial^{0}
$$

In these terms eq. (95) takes the form of a (covariant) harmonic analyticity condition:

$$
\frac{\partial f^{q}}{\partial \bar{y}}+\frac{q y}{2(1+y \bar{y})} f^{q}=0
$$

It admits the general solution $f^{q}(y, \bar{y})=(1+y \bar{y})^{-\frac{q}{2}} f_{0}(y)$ where $f_{0}(y)$ is an arbitrary holomorphic function. Remembering that we are looking for solutions globally defined on the sphere it is not hard to show that for $q<0$ the only solution is $f_{0}=0$ and for $q \geq 0 f_{0}(y)$ must be a polynomial of degree $q$ whose $S U(2)$ covariant form is given in (95).

It should be stressed that the above $\mathrm{H}$-analytic harmonic functions are regular, i.e. well-defined on the whole of $S^{2}$. In practice one has also to deal with singular harmonic functions. A typical example we shall encounter in what follows is the harmonic distribution

$$
\frac{1}{(12)}
$$

where

$$
(12) \equiv u_{1}^{+i} u_{2 i}^{+}=\frac{y_{2}-y_{1}}{\sqrt{\left(1+y_{1} \bar{y}_{1}\right)\left(1+y_{2} \bar{y}_{2}\right)}}
$$

At first sight, it is a function of $u^{+}$only, therefore one would expect $\partial_{1}^{++}(12)^{-1}=0$. However, this distribution is singular at the point $u_{1}=u_{2}$, so it should be differentiated with care:

$$
\begin{aligned}
\partial_{1}^{++} \frac{1}{(12)} & =\left(1+y_{1} \bar{y}_{1}\right)^{3 / 2}\left(1+y_{2} \bar{y}_{2}\right)^{1 / 2} \frac{\partial}{\partial \bar{y}_{1}} \frac{1}{y_{1}-y_{2}} \\
& =\left(1+y_{1} \bar{y}_{1}\right)^{2} i \pi \delta\left(y_{1}-y_{2}\right) \\
& =\left(12^{-}\right) \delta\left(u_{1}, u_{2}\right)
\end{aligned}
$$

where we have used the well-known relation

$$
\frac{\partial}{\partial \bar{y}} \frac{1}{y}=i \pi \delta(y)
$$

Note that the factor $\left(12^{-}\right) \equiv u_{1}^{+i} u_{2 i}^{-}$is needed for keeping the balance of charges on both sides of eq. (102) in the $S(2)$ covariant notation. 
The conclusion from the above discussion is that the condition (95) is, on the one hand, the definition of a highest weight of an $S U(2)$ irrep and, on the other hand, a harmonic analyticity condition on the sphere. This type of condition can easily be supersymmetrised in order to be applied to superfields such as the hypermultiplet $q^{+}(90)$ and we obtain the following operator invariant under Q supersymmetry:

$$
D^{++}=\partial^{++}-2 i \theta^{+\alpha} \bar{\theta}^{+\dot{\alpha}} \partial_{\alpha \dot{\alpha}}
$$

where $\partial_{\alpha \dot{\alpha}} \equiv \sigma_{\alpha \dot{\alpha}}^{\mu} \partial / \partial x^{\mu}$.

Now, let us impose the (supercovariant) H-analyticity condition

$$
D^{++} q^{+}\left(x, \theta^{+}, \bar{\theta}^{+}, u\right)=0 .
$$

Inserting the expansion (90) into eq. (105), we obtain a set of harmonic differential equations which are solved just like eq. (95). The result is the short (on-shell) hypermultiplet

$$
q^{+}=f^{i}(x) u_{i}^{+}+\theta^{+\alpha} \psi_{\alpha}(x)+\bar{\theta}_{\dot{\alpha}}^{+} \bar{\xi}^{\dot{\alpha}}(x)+2 i \theta^{+\alpha} \bar{\theta}^{+\dot{\alpha}} \partial_{\alpha \dot{\alpha}} f^{i}(x) u_{i}^{-}
$$

where all the auxiliary fields have been eliminated and the remaining physical ones put on shell,

$$
\square f^{i}(x)=\not \partial \psi=\not \partial \bar{\xi}=0 \text {. }
$$

So, in the case of the hypermultiplet the combination of G- and $\mathrm{H}$-analyticities results in an on-shell superfield. Note that this result crucially depends on the $U(1)$ charge of the G-analytic superfield. For example, a superfield $L^{++}\left(x, \theta^{+}, \bar{\theta}^{+}, u\right)$ of charge +2 subject to the same Hanalyticity condition

$$
D^{++} L^{++}=0
$$

describes an off-shell multiplet (the linear or tensor multiplet consisting of a triplet of real scalars, a divergenceless real vector, a Majorana spinor and a complex auxiliary field). For charges $\geq+3$ the H-analyticity condition simply cuts off the tail of auxiliary fields without imposing any constraints on the remaining physical fields. On the contrary, for charges $\leq 0$ the condition is too strong and only admits a trivial solution.

A very important observation is that the H-analyticity conditions (105) or (107) admit an equivalent form in terms of the harmonic derivative $\partial^{--}$. Remembering that $\partial^{++}$and $\partial^{--}$are the raising and lowering operators of $S U(2)_{R}$ and that the H-analyticity condition $\partial^{++} f^{q}(u)=0$ defines the highest weight of an $S U(2)_{R}$ irrep of isospin $q / 2$ (dimension $q+1$ ), we immediately see the equivalence relation

$$
\partial^{++} f^{q}(u)=0 \quad \Leftrightarrow \quad\left(\partial^{--}\right)^{q+1} f^{q}(u)=0
$$

(alternatively, it can be derived by inspecting the harmonic expansion (81)). The supersymmetric version of the new form of the $\mathrm{H}$-analyticity condition involves the operator

$$
D^{--}=\partial^{--}-2 i \theta^{-\alpha} \bar{\theta}^{-\dot{\alpha}} \partial_{\alpha \dot{\alpha}}+\theta^{-\alpha} \frac{\partial}{\partial \theta^{+\alpha}}+\bar{\theta}^{-\dot{\alpha}} \frac{\partial}{\partial \bar{\theta}^{+\dot{\alpha}}}
$$

There is a crucial difference between these two conditions, which we shall heavily exploit in what follows. The point is that singular harmonic functions of the type (100) give rise to delta-type 
singularities under $\partial^{++}$(see (102)), whereas they can be differentiated as ordinary functions by $\partial^{--}$, e.g.

$$
\partial^{--} \frac{1}{(12)}=-\frac{\left(1^{-} 2\right)}{(12)^{2}}
$$

The explanation is that in the former case we deal with a derivative of the type $\partial / \partial \bar{y} y^{-1}=i \pi \delta(y)$ and in the latter $\partial / \partial y y^{-1}=-y^{-2}$.

In the rest of this section we shall examine the non-trivial implications of $\mathrm{H}$-analyticity combined with the requirement of superconformal covariance for correlation functions of charge +2 . For that purpose we shall need the superconformal transformation properties of the superfields and operators we have introduced. The transformation law of the harmonic derivatives $D^{++}$and $D^{--}$can be found using Cartan's coset scheme [28] (or checked directly [25]):

$$
\begin{aligned}
\delta D^{++} & =-\Lambda^{++} D^{0} \\
\delta D^{--} & =-\left(D^{--} \Lambda^{++}\right) D^{--}
\end{aligned}
$$

where

$$
D^{++} \Lambda=\Lambda^{++}, \quad D^{++} \Lambda^{++}=0
$$

and

$$
\Lambda=a+k_{\alpha \dot{\alpha}} x^{\alpha \dot{\alpha}}+\lambda^{i j} u_{i}^{+} u_{j}^{-}+4 i\left(\theta^{+\alpha} \eta_{\alpha}^{i}+\bar{\eta}_{\dot{\alpha}}^{i} \bar{\theta}^{+\dot{\alpha}}\right) u_{i}^{-}
$$

is the superconformal weight factor. For completeness, besides the $\mathrm{S}$ supersymmetry parameter $\eta$ we have also included those of dilation $a$, conformal boosts $k^{\mu}$ and $S U(2)_{C} \lambda^{i j}$. Then it is not hard to check that the $\mathrm{H}$-analyticity condition

$$
D^{++} q^{+}=0 \quad \Leftrightarrow \quad\left(D^{--}\right)^{2} q^{+}=0
$$

is covariant if the hypermultiplet transforms with superconformal weight +1 :

$$
\delta q^{+}=-\lambda \cdot \partial q^{+}+\Lambda q^{+}
$$

(here $-\lambda \cdot \partial$ denotes the coordinate transformations). Similarly, the linear multiplet subject to the H-analyticity condition

$$
D^{++} L^{++}=0 \quad \Leftrightarrow \quad\left(D^{--}\right)^{3} L^{++}=0
$$

should have weight +2 :

$$
\delta L^{++}=-\lambda \cdot \partial L^{++}+2 \Lambda L^{++} .
$$




\subsection{Two- and three-point functions}

The simplest example of a two-point function is the hypermultiplet propagator

$$
G^{(1,1)}(1 \mid 2)=\left\langle\tilde{q}^{+}(1) q^{+}(2)\right\rangle
$$

where the superscript $(1,1)$ indicates the $U(1)$ charges at the two points and $\sim$ is a special conjugation on $S^{2}$ preserving G-analyticity [18]. It is defined as the Green's function of the field equation (105):

$$
D_{1}^{++} G^{(1,1)}\left(x_{1}, \theta_{1}^{+}, u_{1} \mid x_{2}, \theta_{2}^{+}, u_{2}\right)=\delta^{4}\left(x_{1}-x_{2}\right)\left(\theta_{1}^{+}-\left(u_{1}^{+} u_{2}^{-}\right) \theta_{2}^{+}\right)^{4}\left(u_{1}^{-} u_{2}^{+}\right) \delta\left(u_{1}, u_{2}\right)
$$

Naturally, like the hypermultiplet superfield $q^{+}$itself, the Green's function should be G-analytic. The right-hand side of eq. (119) is the complete delta-function of the G-analytic superspace (as in eq. (102), the factors $\left(u_{1}^{+} u_{2}^{-}\right)$and $\left(u_{1}^{+} u_{2}^{-}\right)$maintain the balance of $U(1)$ charges). Throughout this paper we assume that all the correlation functions are considered away from the coincident points where they usually have singularities. In this case the right-hand side of eq. (119) just vanishes:

$$
D_{1}^{++} G^{(1,1)}(1 \mid 2)=0 \text { for points } 1 \neq 2 .
$$

The same is of course true if we replace $D_{1}^{++}$by $D_{2}^{++}$. In other words, this two-point function is $\mathrm{H}$-analytic away from the singular point.

Another basic property of the hypermultiplet propagator is superconformal covariance. According to the transformation law (115) of the hypermultiplet itself, the propagator transforms as follows:

$$
\delta G^{(1,1)}(1 \mid 2)=-\lambda \cdot \partial G^{(1,1)}(1 \mid 2)+(\Lambda(1)+\Lambda(2)) G^{(1,1)}(1 \mid 2) .
$$

The combination of $\mathrm{H}$-analyticity and the conformal properties of the propagator allow us to find the explicit expression for $G^{(1,1)}$. We start by examining the leading component of this superfield

$$
g^{(1,1)}\left(x_{12}^{2}, u_{1}, u_{2}\right)=G^{(1,1)}\left(\theta_{1}^{+}=\theta_{2}^{+}=0\right) .
$$

Here we have taken into account translation and Lorentz invariance which tell us that the function must depend on the space-time invariant $x_{12}^{2} \equiv\left(x_{1}-x_{2}\right)^{2}$. In the absence of $\theta^{+}$the harmonic derivative $D^{++} \equiv \partial^{++}$, so the H-analyticity condition $(120)$ simply tells us that $g^{(1,1)}$ must be linear in $u_{1}^{+}$(recall (95)). At the same time it is an $S U(2)$ invariant, so the index $i$ of $u_{1 i}^{+}$must be contracted with the other harmonic variable $u_{2}^{ \pm}$. Given the charges +1 at both points, we conclude that the only such invariant combination of harmonics is $(12) \equiv u_{i}^{+i} u_{2 i}^{+}$. So, $g^{(1,1)}$ is reduced to

$$
g^{(1,1)}=(12) g\left(x_{12}^{2}\right) .
$$

The remaining function $g\left(x_{12}^{2}\right)$ can be most easily determined by making use of the dilation part of the conformal group. The first component of the superfield $q^{+}$is the physical scalar $f^{i}(x)$ which has conformal weight 1 , and so does the leading term in the hypermultiplet propagator. 
The harmonic factor in 123 is weightless, so we conclude that $g=C / x_{12}^{2}$. The constant $C$ can be fixed by comparing with the standard scalar propagator and the result is

$$
g^{(1,1)}=\frac{1}{4 i \pi^{2}} \frac{(12)}{x_{12}^{2}} .
$$

Now, the less trivial part of the determination of the propagator $G^{(1,1)}$ is completing it to a full superfield, i.e. restoring the dependence on $\theta_{1,2}^{+}$. Here we shall use a trick which will prove very useful in the study of the four-point correlator in the next subsection. The two-point function is supposed invariant under Q supersymmetry (88), which acts as a shift of the Grassmann variables:

$$
\left(\theta_{1,2}^{+\alpha, \dot{\alpha}}\right)^{\prime}=\theta_{1,2}^{+\alpha, \dot{\alpha}}+u_{1,2 i}^{+} \epsilon^{i \alpha, \dot{\alpha}} .
$$

It is then clear that by making a finite $\mathrm{Q}$ supersymmetry transformation with parameter

$$
\epsilon^{i \alpha, \dot{\alpha}}=\frac{u_{2}^{+i}}{(12)} \theta_{1}^{+\alpha, \dot{\alpha}}-\frac{u_{1}^{+i}}{(12)} \theta_{2}^{+\alpha, \dot{\alpha}}
$$

we can eliminate both $\theta_{1}^{+}$and $\theta_{2}^{+}$:

$$
\text { Q frame: } \quad\left(\theta_{1}^{+\alpha, \dot{\alpha}}\right)^{\prime}=\left(\theta_{2}^{+\alpha, \dot{\alpha}}\right)^{\prime}=0 .
$$

In this frame the two-point function becomes independent of the Grassmann variables. In other words, it coincides with its leading component (124), $\left.G^{(1,1)}\right|_{Q} \equiv g^{(1,1)}$. Then we can go back to the original frame by performing the same finite $Q$ supersymmetry transformations on the remaining coordinates. ${ }^{3}$ Such a transformation only affects the difference $x_{12}$ and gives

$$
\hat{x}_{12}^{\alpha \dot{\alpha}}=x_{12}^{\alpha \dot{\alpha}}+\frac{4 i}{(12)}\left[\left(1^{-} 2\right) \theta_{1}^{+} \bar{\theta}_{1}^{+}+\left(2^{-} 1\right) \theta_{2}^{+} \bar{\theta}_{2}^{+}+\theta_{1}^{+} \bar{\theta}_{2}^{+}+\theta_{2}^{+} \bar{\theta}_{1}^{+}\right]^{\alpha \dot{\alpha}} .
$$

By construction, this modified coordinate difference is invariant under Q supersymmetry, which can be easily verified using (88). So, to find out the $\theta_{1,2}^{+}$dependence of the hypermultiplet propagator, it is enough to replace $x_{12}$ by $\hat{x}_{12}$ :

$$
G^{(1,1)}(1 \mid 2)=\frac{1}{4 i \pi^{2}} \frac{(12)}{\hat{x}_{12}^{2}}
$$

In deriving this two-point function we have only used the dilation part of the superconformal group. In fact, since the result is unique, it is guaranteed to have the right superconformal properties (121) of the propagator (this can also be checked directly). Further, so far we have only solved the $\mathrm{H}$-analyticity constraint (120) at the lowest (leading) order of the $\theta^{+}$expansion. One might try to argue that since the left-hand side of eq. (120) is itself an invariant of $\mathrm{Q}$ supersymmetry, it is sufficient to check H-analyticity in the Q frame (i.e., in the absence of $\theta^{+}$). However, this argument is not safe here. Indeed, in the expansion of the two-point function there are harmonic singularities of pole type (e.g., $\left.(12)^{-1}\right)$, on which the operator $\partial^{++}$creates a delta-type singularity (recall (102)). In such a situation we will not be allowed to use the

\footnotetext{
${ }^{3}$ As a simpler example of this trick, consider translation invariance for a set of two space-time points $x_{1}, x_{2}$. By means of the finite translation $P: x_{2}^{\prime}=x_{2}+a=0$ we can go to a $P$ frame in which only $x_{1}$ survives. Then, to restore manifest invariance, we make the same shift on $x_{1}: x_{1}^{\prime}=x_{1}+a=x_{1}-x_{2} \equiv x_{12}$.
} 
supersymmetry parameter (126) which itself contains harmonic poles. A safe way to extend $\mathrm{H}$-analyticity to all orders in the $\theta^{+}$expansion by means of the transformation (126) is to use the alternative form of the $\mathrm{H}$-analyticity constraint involving $D^{--}$(see (114)). We shall come back to this important point in the next subsection.

Knowing the hypermultiplet propagator, we can easily predict the general form of correlators of two or three composite operators made out of hypermultiplets. Take, for instance, the two-point function

$$
G^{(2,2)}(1 \mid 2)=\left\langle\operatorname{Tr}\left(\tilde{q}^{+}(1)\right)^{2} \operatorname{Tr}\left(q^{+}(2)\right)^{2}\right\rangle .
$$

Note that it has charges +2 at each point matching the number of elementary hypermultiplets in each composite operator. One can imagine this correlator in the context of $N=4$ super-YangMills theory, where a hypermultiplet in the adjoint representation of the gauge group interacts with the $N=2$ super-Yang-Mills gauge potential. Since the $N=4$ theory is finite (conformally invariant), we can demand that the correlator (130) be superconformally covariant,

$$
\delta G^{(2,2)}(1 \mid 2)=-\lambda \cdot \partial G^{(2,2)}(1 \mid 2)+2(\Lambda(1)+\Lambda(2)) G^{(2,2)}(1 \mid 2) .
$$

In addition to this, the correlator should be $\mathrm{H}$-analytic. Indeed, let us differentiate it with the harmonic derivative $D^{++}$. 1 Since $D^{++}$is the operator of the free field equation (90) for the hypermultiplet, one can argue that such a differentiation will give rise to a Schwinger-Dyson equation for the correlator:

$$
D_{1}^{++} G^{(2,2)}(1 \mid 2)=\text { contact terms . }
$$

Since the composite operators are bilinear in this case (charges +2 ), equation (132) can also be interpreted as a Ward identity. Indeed, the bilinears $\tilde{q}^{+} \tilde{q}^{+}, \tilde{q}^{+} q^{+}$and $q^{+} q^{+}$are the currents of an extra $S U(2)$ symmetry of the $N=4$ theory realised in terms of $N=2$ superfields?. So, in this case eq. (132) corresponds to the current conservation law. In the context of this paper we treat contact terms as zeros, so eq. (132) takes the form of an H-analyticity condition:

$$
D_{1}^{++} G^{(2,2)}(1 \mid 2)=0 \text { for points } 1 \neq 2
$$

(and similarly at point 2). Since the product of two H-analytic functions is $\mathrm{H}$-analytic as well, we immediately find an obvious solution to this constraint as the square of the hypermultiplet propagator,

$$
G^{(2,2)}(1 \mid 2)=C \frac{(12)^{2}}{\hat{x}_{12}^{4}}
$$

where $C$ is a constant. In fact, this is the general solution. The argument is as in the case of the propagator. One first examines the leading component $G^{(2,2)}\left(\theta_{1}^{+}=\theta_{2}^{+}=0\right)$. The constraint (133) fixes the harmonic dependence since the combination $(12)^{2}$ is the only $S U(2)$ invariant of charges $(2,2)$ annihilated by $\partial^{++}$. The dependence on $x_{12}^{2}$ is determined by simple dilation covariance. Finally, with two G-analytic Grassmann variables $\theta_{1,2}^{+}$we already know that the complete $\theta^{+}$dependence is fixed by $\mathrm{Q}$ supersymmetry alone, by just putting a hat on $x_{12}^{2}$. It should be mentioned that the above considerations cannot predict the value of the constant in

\footnotetext{
${ }^{4}$ The traces in $(130)$ make the composite operators gauge invariant, so we can use a flat $D^{++}$(no gauge connection).

${ }^{5}$ In fact, this symmetry is the visible part of the full $S U(4) \mathrm{R}$ symmetry of the $N=4$ theory.
} 
(134). In principle, it might receive quantum corrections at each level of perturbation theory, but it can be shown that this type of correlator is protected by a non-renormalisation theorem [5, 8].

Next we turn to three-point correlators. As an example, take the correlator of three currents, i.e. bilinears made out of hypermultiplets:

$$
G^{(2,2,2)}(1|2| 3)=\left\langle\operatorname{Tr}\left(\tilde{q}^{+}(1)\right)^{2} \operatorname{Tr}\left(\tilde{q}^{+}(2) q^{+}(2)\right) \operatorname{Tr}\left(q^{+}(3)\right)^{2}\right\rangle
$$

and subject to the requirements of H-analyticity

$$
D_{1}^{++} G^{(2,2,2)}(1|2| 3)=0 \text { for points } 1 \neq 2 \neq 3
$$

and of superconformal covariance

$$
\delta G^{(2,2,2)}(1|2| 3)=-\lambda \cdot \partial G^{(2,2,2)}(1|2| 3)+2(\Lambda(1)+\Lambda(2)+\Lambda(3)) G^{(2,2,2)}(1|2| 3) .
$$

Just as for $G^{(2,2)}(1 \mid 2)$ above, it is obvious that the product of three propagators

$$
G^{(2,2,2)}(1|2| 3)=C \frac{(12)}{\hat{x}_{12}^{2}} \frac{(23)}{\hat{x}_{23}^{2}} \frac{(31)}{\hat{x}_{31}^{2}}
$$

satisfies both requirements. To prove its uniqueness, we argue as follows. Firstly, at the lowest level in the $\theta^{+}$expansion there is a single $S U(2)$ invariant combination of the three harmonics with the right charges and vanishing under $D_{1}^{++}$, namely (12)(23)(31). Secondly, the space-time dependence is now determined by the full conformal group (and not just dilations, as for two points). It is well-known that there exists no conformal invariant made out of three space-time variables, therefore the product $x_{12}^{-2} x_{23}^{-2} x_{31}^{-2}$ is the only function with the required conformal properties. Finally, we have to show that putting hats on the $x$ 's gives the unique completion of the leading component to a full superfield. Before we did this by using the Q frame (127) in which the two Grassmann variables had been eliminated. Now we have three $\theta^{+}$'s, and the Q supersymmetry parameter $\epsilon^{i}$ alone is not enough to shift away all of them. This time we have to invoke S supersymmetry as well. Looking at the transformation law of $\theta^{+}$in (89) we see that S supersymmetry acts essentially as a shift (although non-linear), provided that the matrix $x^{\alpha \dot{\alpha}}$ is invertible. Then the combination of $\mathrm{Q}$ and $\mathrm{S}$ supersymmetry makes it possible to find a

$$
\text { Q\&S frame: } \quad\left(\theta_{1}^{+\alpha, \dot{\alpha}}\right)^{\prime}=\left(\theta_{2}^{+\alpha, \dot{\alpha}}\right)^{\prime}=\left(\theta_{3}^{+\alpha, \dot{\alpha}}\right)^{\prime}=0
$$

in which there are no $\theta^{+}$'s left. [. So, if there existed another superfield completion of the leading component above, their difference would be a nilpotent (i.e., proportional to $\theta^{+}$) superconformal covariant. But such an object would vanish in the Q\&S frame, therefore it must vanish in any frame. So, H-analyticity and superconformal covariance can predict the form of the three-point correlator up to a constant factor. Once again, it turns out protected by a non-renormalisation theorem [5, 怔.

\subsection{Four-point correlators}

\subsubsection{Preliminaries}

The main subject of interest in this paper are four-point correlators of hypermultiplet bilinears of the type, e.g.,

\footnotetext{
${ }^{6}$ In fact, the S supersymmetry parameter $\eta^{i}$ is an $S U(2)$ doublet, just as $\epsilon^{i}$. Using both of them we can shift away up to four $\theta^{+}$, as we shall do in the four-point case.
} 


$$
G^{(2,2,2,2)}(1|2| 3 \mid 4)=\left\langle\operatorname{Tr}\left(\tilde{q}^{+}(1)\right)^{2} \operatorname{Tr}\left(q^{+}(2)\right)^{2} \operatorname{Tr}\left(\tilde{q}^{+}(3)\right)^{2} \operatorname{Tr}\left(q^{+}(4)\right)^{2}\right\rangle
$$

satisfying the requirements of $\mathrm{H}$-analyticity

$$
D_{1}^{++} G^{(2,2,2,2)}(1|2| 3 \mid 4)=0 \text { for points } 1 \neq 2 \neq 3 \neq 4
$$

and of superconformal covariance

$$
\delta G^{(2,2,2,2)}(1|2| 3 \mid 4)=-\lambda \cdot \partial G^{(2,2,2,2)}(1|2| 3 \mid 4)+2(\Lambda(1)+\Lambda(2)+\Lambda(3)+\Lambda(4)) G^{(2,2,2,2)}(1|2| 3 \mid 4) .
$$

Compared to the two- and three-point cases above, the structure of the four-point correlator is considerably richer, for two main reasons which can be seen at the lowest level in the $\theta^{+}$ expansion. Firstly, now there exist three independent harmonic combinations satisfying (141):

$$
(12)^{2}(34)^{2},(14)^{2}(23)^{2},(12)(23)(34)(41) \text {. }
$$

Any other combination can be reduced to these by means of the harmonic cyclic identity

$$
(12)(34)+(13)(42)+(14)(23)=0
$$

following from the property of the $\epsilon^{i j}$ contraction. Secondly, given four space-time points, one can construct two independent conformal invariants.] the cross-ratios

$$
s=\frac{x_{14}^{2} x_{23}^{2}}{x_{12}^{2} x_{34}^{2}}, \quad t=\frac{x_{13}^{2} x_{24}^{2}}{x_{12}^{2} x_{34}^{2}} .
$$

Consequently, the most general form of the leading component of the correlator (140) consistent with $\mathrm{H}$-analyticity and conformal covariance is

$$
\frac{(12)^{2}(34)^{2}}{x_{12}^{4} x_{34}^{4}} a(s, t)+\frac{(14)^{2}(23)^{2}}{x_{14}^{4} x_{23}^{4}} b(s, t)+\frac{(12)(23)(34)(41)}{x_{12}^{2} x_{23}^{2} x_{34}^{2} x_{41}^{2}} c(s, t) .
$$

Here we see the three independent harmonic structures (143) completed to product of propagators. Such products already have the required conformal properties of the correlator, so the only freedom left are the three arbitrary coefficient functions $a, b, c$ of the invariant cross-ratios. Our aim will be to find constraints on these functions following from the full implementation of $\mathrm{H}$-analyticity combined with superconformal covariance.

The first step is to argue, just like in the three-point case, that there exists a special frame in superspace in which there are no $\theta^{+}$left:

$$
\text { Q\&S frame: } \quad\left(\theta_{1}^{+\alpha, \dot{\alpha}}\right)^{\prime}=\left(\theta_{2}^{+\alpha, \dot{\alpha}}\right)^{\prime}=\left(\theta_{3}^{+\alpha, \dot{\alpha}}\right)^{\prime}=\left(\theta_{4}^{+\alpha, \dot{\alpha}}\right)^{\prime}=0 .
$$

As explained above, this can be achieved by fully exploiting the four spinor parameters contained in the doublets $\epsilon^{i}$ of $\mathrm{Q}$ and $\eta^{i}$ of $\mathrm{S}$ supersymmetry to shift away all four $\theta^{+}$. The existence of such

\footnotetext{
${ }^{7}$ Here is a simple explanation, very much in the spirit of the Q\&S frame argument above. The translations $P_{\mu}$ and the conformal boosts $K_{\mu}$ act on $x^{\mu}$ as linear and non-linear shifts, correspondingly. So, the combined action of both of them can define a special P\&K frame in which there are only two out of the four space-time variables $x_{1,2,3,4}^{\mu}$ left. Out of them we can make three Lorentz invariants (the two squares and the scalar product). Finally, dilation invariance requires that we take the two independent ratios of those.
} 
a frame implies that the completion of the leading component (146) to a full superfield is always possible and is uniquely determined by $\mathrm{Q}$ and $\mathrm{S}$ supersymmetry. To obtain this completion one could, in principle, find the finite transformation to the frame (147). However, unlike the case of the linear Q supersymmetry, S supersymmetry acts on the coordinates in a very non-linear way and the practical realisation of this step is not at all easy. Fortunately, as we shall explain below, for our purposes we shall only need to know the first non-trivial level in the $\theta^{+}$expansion of the correlator.

The above discussion makes it clear that no further constraints on the coefficient functions $a, b, c$ originate from conformal supersymmetry alone. This only takes place when we try to impose H-analyticity. There are two possible approaches in doing so. One is to use the form (141) of the constraint and try to solve it level by level in the $\theta^{+}$expansion. The problem here is that this expansion is very complicated (assuming that we have already found it, which is in itself not an easy task). A much more efficient approach is to use the alternative form

$$
\left(D_{4}^{--}\right)^{3} G^{(2,2,2,2)}(1|2| 3 \mid 4)=0 \text {. }
$$

The advantage is that we can study this constraint in the Q\&S frame (147) where there are no $\theta^{+}$'s. This results in substantial technical simplifications. The same trick is not allowed in the form (141) because of the harmonic singularities (see the discussion after eq. (129)).

\subsubsection{An example of H-analyticity in the $\mathrm{Q}$ frame}

In order to better understand the idea of this approach, we are going to redo the derivation of the propagator (129), but this time starting form the alternative form of the H-analyticity condition

$$
\left(D_{1}^{--}\right)^{2} G^{(1,1)}(1 \mid 2)=0 .
$$

We begin by going to the $\mathrm{Q}$ frame (127). There the left-hand side of eq. (149) does not depend on $\theta^{+}$but can still depend on $\theta^{-}$. In particular, since the operator $D^{--}$converts $\theta^{+}$into $\theta^{-}$(see $(109)$ ), some terms in the $\theta^{+}$expansion of $G^{(1,1)}$ may survive the transformation (125), (126). Therefore we should proceed in the following order.

Step 1. Expand $G^{(1,1)}$ in $\theta_{1}^{+}$up to the order $\theta_{1}^{+} \bar{\theta}_{1}^{+}$(still in the old frame):

$$
G^{(1,1)}=g^{(1,1)}\left(x_{12}^{2}, u_{1}, u_{2}\right)+\theta_{1}^{+\alpha} \bar{\theta}_{1}^{+\dot{\alpha}} \gamma_{\alpha \dot{\alpha}}^{(-1,1)}\left(x_{12}^{2}, u_{1}, u_{2}\right)+\ldots
$$

There is no need to keep terms containing $\theta_{2}^{+}$or higher orders in $\theta_{1}^{+}$because they cannot be "rescued" by $\left(D_{1}^{--}\right)^{2}$ and will vanish after the transformation to the $\mathrm{Q}$ frame. Indeed, the function $G^{(1,1)}$ carries no $\mathrm{R}$ weight, so the Grassmann variables have to appear in its expansion in pairs $\theta^{+} \bar{\theta}^{+}$. So, only the term $\theta_{1}^{+} \bar{\theta}_{1}^{+} \Rightarrow \theta_{1}^{-} \bar{\theta}_{1}^{-}$can survive in the $\mathrm{Q}$ frame.

Step 2. Differentiate the expansion (150) with $\left(D_{1}^{--}\right)^{2}$ keeping only terms without any $\theta^{+}$. The expansion of $\left(D_{1}^{--}\right)^{2}$ is

$$
\left(D_{1}^{--}\right)^{2}=\left(\partial_{1}^{--}\right)^{2}-4 i \theta_{1}^{-} \not \partial_{1} \bar{\theta}_{1}^{-} \partial_{1}^{--}+\left(\theta_{1}^{-} \partial_{1}^{-}+\bar{\theta}_{1}^{-} \bar{\partial}_{1}^{-}\right)^{2}-2\left(\theta_{1}^{-}\right)^{2}\left(\bar{\theta}_{1}^{-}\right)^{2} \square_{1}
$$

We have dropped the terms linear in $2 \theta_{1}^{-} \partial_{1}^{-}+\bar{\theta}_{1}^{-} \bar{\partial}_{1}^{-}$because they only convert one $\theta_{1}^{+}$into $\theta_{1}^{-}$, and the remaining $\theta_{1}^{+}$in the bilinear combination will vanish in the $\mathrm{Q}$ frame. When applied to (150), this operator gives 


$$
\begin{aligned}
\left(D_{1}^{--}\right)^{2} G^{(1,1)}= & \left(\partial_{1}^{--}\right)^{2} g^{(1,1)} \\
& +\theta_{1}^{-\alpha} \bar{\theta}_{1}^{-\dot{\alpha}}\left[2 \gamma_{\alpha \dot{\alpha}}^{(-1,1)}-4 i \partial_{1 \alpha \dot{\alpha}} \partial_{1}^{--} g^{(1,1)}\right] \\
& -2\left(\theta_{1}^{-}\right)^{2}\left(\bar{\theta}_{1}^{-}\right)^{2} \square_{1} g^{(1,1)} \\
& +\theta^{+} \text {terms } \\
= & 0 .
\end{aligned}
$$

Step 3. Make the transformation to the Q frame. The left-hand side of eq. (152) is superconformally covariant, so it is multiplied by the weight factor $\Lambda(1)+\Lambda(2)$. Since it is supposed to vanish, this transformation just amounts to neglecting all the $\theta^{+}$dependence (already taken into account at steps 1 and 2).

The resulting constraint (152) involves three levels in its $\theta^{-}$expansion.

Level 0 or $\left(\theta^{-} \bar{\theta}^{-}\right)^{0}$ :

$$
\left(\partial_{1}^{--}\right)^{2} g^{(1,1)}\left(x_{12}^{2}, u_{1}, u_{2}\right)=0 \quad \Rightarrow \quad g^{(1,1)}=(12) g\left(x_{12}^{2}\right) .
$$

This constraint uniquely fixes the harmonic dependence of the component $g^{(1,1)}$. The combination of harmonics (12) $\equiv u_{1}^{+i} u_{2 i}^{+}$is the only one which is $S U(2)$ invariant, has the right charges and is annihilated by the lowering operator $\left(\partial_{1}^{--}\right)^{2}$.

Level 1 or $\left(\theta^{-} \bar{\theta}^{-}\right)^{1}$ :

$$
\gamma_{\alpha \dot{\alpha}}^{(-1,1)}\left(x_{12}^{2}, u_{1}, u_{2}\right)=2 i \partial_{1 \alpha \dot{\alpha}} \partial_{1}^{--} g^{(1,1)}\left(x_{12}^{2}, u_{1}, u_{2}\right)=2 i(12) \partial_{1 \alpha \dot{\alpha}} g\left(x_{12}^{2}\right) .
$$

Level 2 or $\left(\theta^{-} \bar{\theta}^{-}\right)^{2}$ :

$$
\square_{1} g^{(1,1)}\left(x_{12}^{2}, u_{1}, u_{2}\right)=0 \Rightarrow \square_{1} g\left(x_{12}^{2}\right)=0 \quad \Rightarrow \quad g\left(x_{12}^{2}\right)=\frac{C}{x_{12}^{2}}
$$

where $C$ is an arbitrary constant. We recall that we are only interested in the two-point function away from the coincident point, so we can drop the delta-function $\delta\left(x_{12}\right)$ in (155).

We should mention that in this example we have made no use of conformal invariance or S supersymmetry. Actually, this two-point function is in a sense overdetermined. We have already seen that by imposing $\mathrm{H}$-analyticity just at level 0 and then invoking dilation covariance (part of the conformal symmetry), we arrived at the same result. This, however, is an exceptional property of the propagator (the charges +1 two-point function). The typical situation is illustrated by the charges +2 two-point function (134). It is not hard to show that it remains H-analytic to all orders in the $\theta$ expansion even if we replace the denominator by any function of $\hat{x}_{12}^{2}$. So, its form cannot be determined without some extra input (dilation covariance in this case). The explanation of this fact can be traced back to the different implications of $\mathrm{H}$-analyticity for superfields of charges +1 and +2 : for the former it is an on-shell condition and for the latter an off-shell one (see (105), (107)).

Let us summarise the above example. Using the $\mathrm{Q}$ frame we have been able to solve the $\mathrm{H}$ analyticity constraint (149) to all relevant orders in the $\theta$ expansion. In the process we only used the $\theta^{+}$expansion of the two-point function $G^{(1,1)}$ to the first non-trivial order (level 1) (see $(\overline{150}))$. At no point we encountered delta-type harmonic singularities which cannot coexist with the singular nature of the transformation to the $\mathrm{Q}$ frame. On the contrary, starting with the form (120), we would have to find out the $\theta^{+}$expansion of $G^{(1,1)}$ to all orders (in this case it 
can go up to level 4) and then solve the H-analyticity constraint order by order. The technical advantages of the use of the alternative form of the H-analyticity constraint and of the Q (or Q\&S) frame result in major simplifications in the case of the four-point function.

\subsubsection{Superconformal covariance at level 1}

Now we come back to the four-point function (140). Eq. (146) represents the solution to the Hanalyticity constraint and to the conformal covariance condition at level 0 . We have also argued that the possibility to go to the Q\&S frame (147) guarantees the existence of a unique completion of this level 0 component to a full superfield. The way to find this completion consists of two steps. The first is to put hats on all the $x$ 's in the denominators, thus reconstructing the products of full propagators. We already know that such products have the required superconformal properties of the correlator. The second step is to complete the conformal cross-ratios $s$ and $t$ in the coefficient functions $a, b, c$ to full superconformal invariants $\hat{s}$ and $\hat{t}$. Then the full correlator consistent with superconformal symmetry will have the form

$$
G^{(2,2,2,2)}(1|2| 3 \mid 4)=\frac{(12)^{2}(34)^{2}}{\hat{x}_{12}^{4} \hat{x}_{34}^{4}} a(\hat{s}, \hat{t})+\frac{(14)^{2}(23)^{2}}{\hat{x}_{14}^{4} \hat{x}_{23}^{4}} b(\hat{s}, \hat{t})+\frac{(12)(23)(34)(41)}{\hat{x}_{12}^{2} \hat{x}_{23}^{2} \hat{x}_{34}^{2} \hat{x}_{41}^{2}} c(\hat{s}, \hat{t}) .
$$

To find $\hat{s}$ and $\hat{t}$ to all orders in the four $\theta^{+}$'s is a very non-trivial task (the expansion goes up to level 8, although Q supersymmetry helps bring it down to level 4). Fortunately, the example above has taught us that we only need one level 1 term. So, we are looking for $\hat{s}$ in the form

$$
\hat{s}=s+\sum_{a, b=1}^{4} \theta_{a}^{+\alpha} S_{a b \alpha \dot{\alpha}} \bar{\theta}_{b}^{+\dot{\alpha}}+O\left(\left(\theta^{+} \bar{\theta}^{+}\right)^{2}\right) .
$$

What we really need is just the coefficient $S_{44 \alpha \dot{\alpha}}$. Indeed, although the three derivatives $\left(D_{4}^{--}\right)^{3}$ in (148) can convert a maximum of three $\theta_{4}^{+}$into $\theta_{4}^{-}$, only the term $\theta_{4}^{+} \bar{\theta}_{4}^{+} \Rightarrow \theta_{4}^{-} \bar{\theta}_{4}^{-}$can survive in the Q\&S frame.

The coefficient $S_{44 \alpha \dot{\alpha}}$ can be solved for from a set of linear equations. It is obtained by performing a combined Q (88) and $\mathrm{S}(89)$ supersymmetry transformation on $\hat{s}$ and demanding that it be invariant. In doing so we shall only keep the terms linear in $\bar{\theta}_{4}^{+}$since only they involve the coefficients $S_{a 4}$ :

$$
\delta_{Q+S} \hat{s}=0 \Rightarrow\left[4 i s\left(\epsilon_{4}^{-\alpha}+x_{4}^{\alpha \dot{\beta}} \bar{\eta}_{4 \dot{\beta}}^{-}\right)\left(\frac{x_{14}}{x_{14}^{2}}-\frac{x_{34}}{x_{34}^{2}}\right)_{\alpha \dot{\alpha}}+\sum_{a=1}^{4}\left(\epsilon_{a}^{+\alpha}+x_{a}^{\alpha \dot{\beta}} \bar{\eta}_{a \dot{\beta}}^{+}\right) S_{a 4 \alpha \dot{\alpha}}\right] \bar{\theta}_{4}^{+\dot{\alpha}}=0
$$

Here $(\epsilon, \eta)_{a}^{ \pm} \equiv u_{a i}^{ \pm}(\epsilon, \eta)^{i}$. Removing $\bar{\theta}_{4}^{+\dot{\alpha}}$ and the independent parameters $\epsilon, \eta$ from (158), we obtain four linear equations (one for each harmonic projection of the two parameters) for the four coefficients $S_{a 4}$ :

$$
\begin{aligned}
& 4 i s X_{2}-\sum_{a=1}^{3}(a 4) S_{a 4}=0, \quad \sum_{a=1}^{4}\left(4^{-} a\right) S_{a 4}=0, \\
& (12) x_{24} S_{24}+(13) x_{34} S_{34}=0, \quad(21) x_{14} S_{14}+(23) x_{34} S_{34}=0 .
\end{aligned}
$$


Here and in what follows we use the vectors

$$
X_{1}=\frac{x_{14}}{x_{14}^{2}}-\frac{x_{24}}{x_{24}^{2}}, \quad X_{2}=\frac{x_{14}}{x_{14}^{2}}-\frac{x_{34}}{x_{34}^{2}}, \quad X_{3}=\frac{x_{34}}{x_{34}^{2}}-\frac{x_{24}}{x_{24}^{2}}
$$

having the useful properties

$$
\frac{X_{1}^{2}}{X_{3}^{2}}=\frac{1}{s}, \quad \frac{X_{2}^{2}}{X_{3}^{2}}=\frac{t}{s}, \quad \frac{2 X_{1} \cdot X_{3}}{X_{3}^{2}}=\frac{1+s-t}{s}, \quad \frac{2 X_{2} \cdot X_{3}}{X_{3}^{2}}=\frac{1-s-t}{s} .
$$

Solving eqs. (159) is a straightforward calculation. The result for the coefficient $S_{44}$ is

$$
\begin{aligned}
\frac{(12)^{2}(34)^{2} Y}{4 i} S_{44} & =-(12)(13)(23) t X_{3} \\
& -\left[(12)(13)(23) s+(12)^{2}(34)\left(34^{-}\right) s\right. \\
& \left.+(12)(23)(34)\left(14^{-}\right)(1+s-t)+(14)\left(14^{-}\right)(23)^{2}\right] X_{2}
\end{aligned}
$$

where

$$
Y=1+\frac{1+s-t}{s} U+\frac{1}{s} U^{2}, \quad U=\frac{(14)(23)}{(12)(34)} .
$$

Note that the vector (162) can be rewritten in the equivalent form

$$
\frac{(12)^{2}(34)^{2} Y}{4 i} S_{44}=-\frac{s}{2} \partial_{4}^{--}\left[(12)^{2}(34)^{2} Y\right] X_{2}+(12)(13)(23)\left[-t X_{3}+\frac{1}{2}(1-s-t) X_{2}\right] .
$$

In in a similar manner we can find the relevant term in the expansion of $\hat{t}$

$$
\hat{t}=t+\sum_{a, b=1}^{4} \theta_{a}^{+\alpha} T_{a b \alpha \dot{\alpha}} \bar{\theta}_{b}^{+\dot{\alpha}}+\ldots
$$

The result for $T_{44}$ can be easily obtained from (162) by exchanging $1 \leftrightarrow 2$ which implies $s \leftrightarrow t$, $X_{1} \rightarrow-X_{1}, X_{2} \rightarrow-X_{3}, Y \rightarrow s / t Y$.

The expansion of $\hat{s}, \hat{t}$ is not the only source of $\theta_{4}^{+} \bar{\theta}_{4}^{+}$terms. Another contribution comes from expanding $\hat{x}_{a 4}^{2}, a=1,2,3$ in the propagators in (156):

$$
\hat{x}_{a 4}^{2}=x_{a 4}^{2}-4 i \frac{\left(a 4^{-}\right)}{(a 4)} \theta_{4}^{+} x_{a 4} \bar{\theta}_{4}^{+}+\ldots
$$

(recall (128)). Collecting all of these contributions, we can write down

$$
\begin{aligned}
G^{(2,2,2,2)}(1|2| 3 \mid 4) & \equiv \Pi\left(\hat{x}_{a b}^{2}\right) \cdot f(\hat{s}, \hat{t}) \\
& =\left.G^{(2,2,2,2)}\right|_{\text {level } 0} \\
& +\theta_{4}^{+}\left[-4 i \sum_{a=1}^{3} \frac{\left(a 4^{-}\right)}{(a 4)} x_{a 4} \frac{\partial \Pi}{\partial x_{a 4}^{2}} \cdot f+\Pi \cdot\left(f_{s} S_{44}+f_{t} T_{44}\right)\right] \bar{\theta}_{4}^{+}+\ldots
\end{aligned}
$$


Here $\Pi \cdot f$ is a shorthand for the product of propagators $(\Pi)$ and coefficient functions $(f)$ in (156) and $f_{s, t}=\partial f / \partial(s, t)$. This accomplishes Step 1 of our programme for imposing H-analyticity in the form (148).

\subsubsection{Constraints from H-analyticity}

Step 2 consists of differentiating the expression $(167)$ with the operator $\left(D_{4}^{--}\right)^{3}$

$$
\begin{aligned}
\left(D_{1}^{--}\right)^{3} & =\left(\partial_{1}^{--}\right)^{3} \\
& +3 \partial_{1}^{--}\left(\theta_{1}^{-} \partial_{1}^{-}+\bar{\theta}_{1}^{-} \bar{\partial}_{1}^{-}\right)^{2}-6 i\left(\partial_{1}^{--}\right)^{2} \theta_{1}^{-} \not_{1} \bar{\theta}_{1}^{-} \\
& -6 i \theta_{1}^{-} \not_{1} \bar{\theta}_{1}^{-}\left(\theta_{1}^{-} \partial_{1}^{-}+\bar{\theta}_{1}^{-} \bar{\partial}_{1}^{-}\right)^{2}-6 \partial_{1}^{--}\left(\theta_{1}^{-}\right)^{2}\left(\bar{\theta}_{1}^{-}\right)^{2} \square_{1} \\
& + \text { irrelevant terms }
\end{aligned}
$$

The irrelevant terms in (168) are those which convert an odd number of $\theta_{1}^{+}$into $\theta_{1}^{-}$and thus disappear in the $\mathrm{Q}$ frame. We then apply (168) to (167) and collect all the terms at levels 0 , 1 and 2. In fact, we have already solved the $\mathrm{H}$-analyticity constraint at level 0 in (146). So, it remains to examine the constraints at levels 1 and 2. It is not hard to see that they take the form

Level 1: $\quad \partial_{4}^{--} A^{\mu}=0$,

Level 2: $\quad \partial_{4 \mu} A^{\mu}=0$

where

$$
A^{\mu}=-i \partial_{4}^{--} \partial_{4}^{\mu}(\Pi \cdot f)-4 i \sum_{a=1}^{3} \frac{\left(a 4^{-}\right)}{(a 4)} x_{a 4}^{\mu} \frac{\partial \Pi}{\partial x_{a 4}^{2}} \cdot f+\Pi \cdot\left(f_{s} S_{44}^{\mu}+f_{t} T_{44}^{\mu}\right)
$$

After some simple algebra and using the relations

$$
\partial_{4}^{\mu} s=-2 s X_{2}^{\mu}, \quad \partial_{4}^{\mu} t=2 t X_{3}^{\mu},
$$

we obtain

$$
A^{\mu}=\frac{2 i c}{s} \frac{(12)(13)(23)}{x_{12}^{4} x_{34}^{4}} X_{2}^{\mu}+\left[2 i s X_{2}^{\mu} \partial_{4}^{--} \Pi+S_{44}^{\mu} \Pi\right] \cdot f_{s}+\left[-2 i t X_{3}^{\mu} \partial_{4}^{--} \Pi+T_{44}^{\mu} \Pi\right] \cdot f_{t} .
$$

The terms in the brackets are computed with the help of (164), e.g.

$$
\begin{aligned}
{\left[2 i s X_{2} \partial_{4}^{--} \Pi+S_{44} \Pi\right] \cdot f_{s} } & =2 i s X_{2}(12)^{2}(34)^{2} Y \partial_{4}^{--}\left(\frac{\Pi \cdot f_{s}}{(12)^{2}(34)^{2} Y}\right) \\
& +4 i(12)(13)(23)\left[-t X_{3}+\frac{1}{2}(1-s-t) X_{2}\right] \frac{\Pi \cdot f_{s}}{(12)^{2}(34)^{2} Y}
\end{aligned}
$$

Further, it is convenient to use the harmonic cross-ratio $U$ introduced in (163) and rewrite 


$$
\frac{\Pi \cdot f_{s}}{(12)^{2}(34)^{2} Y}=\frac{1}{x_{12}^{4} x_{34}^{4}} \frac{a_{s}-\frac{c_{s}}{s} U+\frac{b_{s}}{s^{2}} U^{2}}{1+\frac{1+s-t}{s} U+\frac{1}{s} U^{2}},
$$

after which the harmonic derivative $\partial_{4}^{--}$in $(174)$ can be computed using the identity

$$
\partial_{4}^{--} U=-\frac{(12)(13)(23)}{(12)^{2}(34)^{2}}
$$

Repeating the same procedure for the other bracket in $(173)$ and collecting all the terms proportional to the vector $X_{2}$, we obtain the following contribution to the vector $A^{\mu}$ :

$$
2 i \frac{(12)(13)(23)}{x_{12}^{4} x_{34}^{4}}\left[\frac{c}{s}+\beta_{0} \frac{1+\frac{\beta_{1}}{\beta_{0}} U+\frac{\beta_{2}}{\beta_{0}} U^{2}}{1+\frac{1+s-t}{s} U+\frac{1}{s} U^{2}}\right] X_{2}^{\mu}
$$

where

$$
\begin{aligned}
& \beta_{0}=c_{s}+2(1-t) a_{s}-2 t a_{t}, \\
& \beta_{1}=2 a_{s}-\frac{2}{s} b_{s}+\frac{2 t}{s} c_{t}+\frac{s+t-1}{s} c_{s}, \\
& \beta_{2}=-\frac{2}{s} b_{s}-\frac{2 t}{s^{2}} b_{t}-\frac{1}{s} c_{s} .
\end{aligned}
$$

We still have to compute the $X_{3}$ contribution to $A^{\mu}$, but even before this we can already impose the level 1 constraint (169) on the $X_{2}$ contribution (the vectors $X_{2}$ and $X_{3}$ are linearly independent and $\partial_{4}^{--} X_{2}=\partial_{4}^{--} X_{3}=0$ ). The first term in (176) does not depend on $u_{4}$. The second term is the ratio of two polynomials of degree 2 in the cross-ratio $U$. It is easy to see that its derivative vanishes only if the two polynomials are equal,

$$
1+\frac{\beta_{1}}{\beta_{0}} U+\frac{\beta_{2}}{\beta_{0}} U^{2}=1+\frac{1+s-t}{s} U+\frac{1}{s} U^{2}
$$

Comparing the coefficients in front of $U$ and $U^{2}$, we obtain the following constraints:

$$
\begin{aligned}
& c_{s}=(t-1) a_{s}+t a_{t}-b_{s}-\frac{t}{s} b_{t}, \\
& c_{t}=-s a_{s}-s a_{t}+b_{s}+\frac{t-1}{s} b_{t}
\end{aligned}
$$

constituting our main result. This is a set of two linear first-order partial differential equations for the three coefficient functions $a, b, c$. These equations can only determine two of the three functions. Indeed, let us make the change of variables

$$
a=\alpha+\gamma+\frac{1}{s} b, \quad c=-s \gamma+\frac{t-s-1}{s} b,
$$

after which (179) becomes 


$$
\begin{aligned}
& \gamma_{s}=-\alpha_{s}-\alpha_{t}, \\
& t \gamma_{t}+\gamma=s \alpha_{s}+(s-1) \alpha_{t}
\end{aligned}
$$

and we see that $b$ has dropped out. The set of first-order coupled differential equations (181) can be equivalently rewritten as a set of second-order independent ones:

$$
\begin{aligned}
s \alpha_{s s}+t \alpha_{t t}+(s+t-1) \alpha_{s t}+2\left(\alpha_{s}+\alpha_{t}\right) & =0 \\
s \gamma_{s s}+t \gamma_{t t}+(s+t-1) \gamma_{s t}+2\left(\gamma_{s}+\gamma_{t}\right) & =0 .
\end{aligned}
$$

These constraints are the same as eqs. (79) obtained in section 2 in the coordinate approach.

To compute the contribution to $A^{\mu}$ proportional to $X_{3}$ we go through the same steps. This time we do not find any new constraints. The final form of the vector $A^{\mu}$ is

$$
A^{\mu}=2 i(12)(13)(23)\left(A \frac{X_{2}^{\mu}}{x_{12}^{4} x_{34}^{4} s}+B \frac{X_{3}^{\mu}}{x_{12}^{4} x_{34}^{4} t}\right)
$$

where

$$
A=s c_{s}+2(1-t) s a_{s}-2 s t a_{t}+c, \quad B=-\frac{t^{2}}{s}\left(c_{t}+2 s a_{s}+2 s a_{t}\right) .
$$

The remaining step is to impose the level 2 constraint (170). This is facilitated by the useful property

$$
\partial_{4 \mu}\left(\frac{X_{2}^{\mu}}{x_{12}^{4} x_{34}^{4} s}\right)=\partial_{4 \mu}\left(\frac{X_{3}^{\mu}}{x_{12}^{4} x_{34}^{4} t}\right)=0
$$

of the basis vectors in (183), so we only have to differentiate the scalar coefficients $A$ and $B$. Using the identities (172) and (161), we obtain the constraint

$$
\partial_{4 \mu} A^{\mu}=0 \Rightarrow-\frac{t}{s} A_{s}-B_{t}+\frac{1-s-t}{2 s}\left(\frac{t}{s} A_{t}+\frac{s}{t} B_{s}\right)=0 .
$$

Making the change of variables (180) and after some algebra we discover that this is a corollary of the second-order differential equations (182). So, level 2 does not give rise to any new constraints.

\section{Conclusions}

We see that using either method of analysing analyticity for the four-point charge 2 correlator leads to the same result: The requirements of $\mathrm{H}$-analyticity and superconformal covariance yield constraints which fix the form of the four-point correlator (140) up to an arbitrary function of the conformal cross-ratios. An interesting solution to these constraints is provided by the explicit computation of the correlator at two loops carried out in [4, 13]. The result for the level 0 component is

$$
\Phi(s, t)\left[\frac{(12)^{2}(34)^{2}}{x_{12}^{4} x_{34}^{4}}+\frac{(14)^{2}(23)^{2}}{x_{14}^{4} x_{23}^{4}} s+\frac{(12)(23)(34)(41)}{x_{12}^{2} x_{23}^{2} x_{34}^{2} x_{41}^{2}}(t-s-1)\right] .
$$


Here $\Phi(s, t)=\Phi(t, s)=\frac{1}{s} \Phi\left(\frac{1}{s}, \frac{t}{s}\right)$ is a function given by the one-loop scalar box integral. This solution is symmetric under the exchange $1 \leftrightarrow 3$ and is determined by the asymptotic behaviour $\lim _{x_{14} \rightarrow 0} c(s, t)=0$ (see 4 for details). It is interesting to note how the result (186) was obtained: the two-loop calculation in 顿 only provided us with the explicit form of the first two terms in (186); the third term was given as a complicated two-loop integral; the subsequent use of the differential equations (182) in conjunction with the boundary conditions following from the known asymptotic behaviour of the two-loop integral allowed us [13] to solve for the third term as in (186).

We have shown for a number of cases that harmonic analyticity at all orders in an expansion of a correlator in the odd variables is assured by the constraints arising from the lowest and the linear order. We conjecture that this is a general feature.

Acknowledgements: This work was supported in part by the British-French scientific programme Alliance (project 98074), by the EU network on Integrability, non-perturbative effects and symmetry in quantum field theory (FMRX-CT96-0012) and by the grant INTAS-96-0308.

\section{References}

[1] J. Maldacena, The large $N$ limit of superconformal field theories and supergravity, Adv. Theor. Math. Phys. 2 (1998) 231-252, hep-th/9711200; S.S. Gubser, I.R. Klebanov and A.M. Polyakov, Gauge theory correlators from noncritical String theory, Phys. Lett. B428 (1998) 105, hep-th/9802109; E. Witten, Anti-de Sitter space and holography, Adv. Theor. Math. Phys. 2 (1998) 253-291, hep-th/9802150.

[2] Hong-Liu and A.A. Tseytlin, On four-point functions in the CFT/AdS correspondence, hep-th/9807097; D.Z. Freedman, S. Mathur, A. Matusis and L. Rastelli, Comments on four-points functions in the CFT/AdS correspondence, hep-th/9808006; E. d' Hoker and D.Z. Freedman, Gauge boson exchange in AdS $(d+1)$, hep-th/9809179; E. D'Hoker, D.Z. Freedman, S.D. Mathur, A. Matusis, L. Rastelli, Graviton exchange and complete four point functions in the AdS / CFT correspondence hep-th/9903196; E. D'Hoker, D.Z. Freedman, L. Rastelli, AdS / CFT four point functions: How to succeed at z integrals without really trying hep-th/9905049.

[3] M. Bianchi, M. Green, S. Kovacs and G. Rossi, Instantons in supersymmetric Yang-Mills and D instantons in IIB superstring theory, JHEP 08 (1998) 13, hep-th/9807033; N. Dorey, T. Hollowood, V. Khoze, M. Mattis and S. Vandoren, Mulitinstantons and Maldacena's conjecture hep-th/9811060; F. Gonzalez-Rey, I. Park and K. Schalm, A note on four-point functions of conformal operators in $N=4$ super-Yang Mills, Phys. Lett. B448 (1999) 37-40, hep-th/9811155;

[4] B. Eden, P.S. Howe, C. Schubert, E. Sokatchev and P.C. West, Four-point functions in N=4 supersymmetric Yang-Mills theory at two loops, Nucl. Phys. B557 (1999) 355-379, hep-th/9811172.

[5] P.S. Howe and P.C. West, Non-perturbative Green's functions in theories with extended superconformal symmetry, hep-th/9509140; OPEs in four-dimensional superconformal field 
theories, Phys. Lett. B389 (1996) 273-279, hep-th/9607060; Is $N=4$ Yang-Mills soluble? (1996) in Moscow 1996 Physics, 622-626, hep-th/9611074.

[6] P.S. Howe and P.C. West, Superconformal invariants and extended supersymmetry, Phys. Lett. B400 (1997) 307-313, hep-th/9611075.

[7] B. Conlong and P. West, Anomalous dimensions of fields in a supersymmetric quantum field theory at a renormalization group fixed point, J. Phys. A26 (1993) 3325; H. Osborn, $N=1$ superconformal symmetry in four-dimensional quantum field theory Ann. Phys. (N.Y.) 272 (1999) 243-294, hep-th/980804; J-H. Park, Superconformal symmetry and correlation functions hep-th/9903230.

[8] P.S. Howe, E. Sokatchev and P.C. West, Three-point functions in N=4 Yang-Mills, Phys. Lett. B444 (1998) 341-351, hep-th/9808162.

[9] S. Lee, S. Minwalla, M. Rangamani and N. Seiberg, Three-point functions of chiral operators in $D=4, N=4 S Y M$ at large N, Adv. Theor. Math. Phys 2 (1998) 697-718, hep-th/9806074; F. Gonzalez-Rey, B. Kulik and I.Y. Park, Non-renormalisation of two and three point correlators of N=4 SYM in N=1 superspace, Phys. Lett. B455 (1999) 164-170, hep-th/9903094; S. Penati, A. Santambrogio and D. Zanon, Two-point functions of chiral operators in $N=4$ SYM at order $g^{4}$, Bicocca-FT-99-30, hep-th/9910197.

[10] D. Z. Freedman, S.D. Mathur, A. Matusis and L. Rastelli, Correlation functions in the $C F T(d) / A d S(d+1)$ correspondence, Nucl. Phys. B546 (1999) 96-118, hep-th/9804058.

[11] E. D'Hoker, D.Z. Freedman and W. Skiba, Field theory tests for correlators in the AdS/CFT correspondence, Phys. Rev. D59 (1999) 45008, hep-th/9807098.

[12] K. Intriligator, Bonus symmetries of $N=4$ super-Yang-Mills correlation functions via AdS duality, Nucl. Phys. B551 (1999) 575-600, hep-th/9811047.

[13] B. Eden, P.S. Howe, C. Schubert, E. Sokatchev and P.C. West, Simplifications of four-point functions in $N=4$ supersymmetric Yang-Mills theory at two loops, Phys. Lett. B466 (1999) 20-26, hep-th/9906051.

[14] B. Eden, P.S. Howe, C. Schubert, E. Sokatchev and P.C. West, Extremal correlators in four-dimensional SCFT, Phys. Lett. B472 (2000) 323-331, hep-th/9910150.

[15] E. D'Hoker, D.Z. Freedman, S. D. Mathur, A. Matusis and L. Rastelli, Extremal correlators in the AdS/CFT correspondence, MIT-CTP-2893, hep-th/9908160.

[16] G. Arutyunov and S. Frolov, Some cubic couplings in type IIB supergravity on $A d S_{5} \times S^{5}$ and three-point functions in $S Y M_{4}$ at large $N$, LMU-TPW 99-12, hep-th/9907085; Scalar quartic couplings in type IIB supergravity on $A d S_{5} \times S^{5}$, LMU-TPW 99-23, hep-th/9912210.

[17] M. Bianchi and S. Kovacs, Nonrenormalization of extremal correlators in $N=4 S Y M$ theory, ROM2F-99-31, hep-th/9910016.

[18] A. Galperin, E. Ivanov, S. Kalitzin, V. Ogievetsky and E. Sokatchev, Unconstrained $N=2$ matter, Yang-Mills and supergravity theories in harmonic superspace, Class. Quant. Grav. 1 (1984) 469. 
[19] G.G. Hartwell and P.S. Howe (N, p, q) harmonic superspace Int. J. Mod. Phys. A10 (1995) 3901-3920, hep-th/9412147; A superspace survey, Class. Quant. Grav. 12 (1995) 1823-1880.

[20] L. Andrianopoli and S. Ferrara, $K-K$ excitations on $A d S_{5} \times S^{5}$ as $N=4$ "primary" superfields, Phys. Lett. B430 (1998) 248-253, hep-th/9803171.

[21] B. Eden, P.S. Howe and P.C. West, Nilpotent invariants in N=4 SYM, Phys. Lett. B463 (1999) 19-26, hep-th/9905085.

[22] P.S. Howe, C. Schubert, E. Sokatchev and P.C. West, Explicit construction of nilpotent covariants in $N=4 S Y M$, KCL-MTH-99-41, hep-th/9910011.

[23] A. Petkou and K. Skenderis, A non-renormalisation theorem for conformal anomalies, hepth/9906030.

[24] A. Galperin, E. Ivanov, V. Ogievetsky and E. Sokatchev, Harmonic supergraphs: Green functions, Class. Quant. Grav. 2 (1985) 601-616; Harmonic supergraphs: Feynman rules and examples, Class. Quant. Grav. 2 (1985) 617-630.

[25] A. Galperin, E. Ivanov, V. Ogievetsky and E. Sokatchev, Conformal invariance in harmonic superspace, preprint JINR E2-85-363 (1985) published in "Quantum Field theory and Quantum Statistics", vol.2, 233-248, A.Hilger, Bristol (1987).

[26] K. Intriligator and W. Skiba, Bonus symmetry and the operator product expansion of $N=4$ Super-Yang-Mills, hep-th/9905020.

[27] P.S. Howe, K.S. Stelle and P.C. West, $N=1 d=6$ harmonic superspace, Class. Quant. Grav. 2 (1985), 815-821; K.S. Stelle, Manifest realizations of extended supersymmetry, Santa Barbara preprint NSF-ITP-85-001.

[28] A. Galperin, E. Ivanov, V. Ogievetsky and E. Sokatchev, Harmonic superspace, CUP, to appear. 